\title{
Nonlinear diffusion in transparent media: the resolvent equation
}

\author{
Lorenzo Giacomelli Salvador Moll Francesco Petitta
}

\begin{abstract}
We consider the partial differential equation

$$
u-f=\operatorname{div}\left(u^{m} \frac{\nabla u}{|\nabla u|}\right)
$$

with $f$ nonnegative and bounded and $m \in \mathbb{R}$. We prove existence and uniqueness of solutions for both the Dirichlet problem (with bounded and nonnegative boundary datum) and the homogeneous Neumann problem. Solutions, which a priori belong to a space of truncated bounded variation functions, are shown to have zero jump part with respect to the $\mathcal{H}^{N-1}$ Hausdorff measure. Results and proofs extend to more general nonlinearities.
\end{abstract}

Keywords. Total Variation, Transparent Media, Linear Growth Lagrangian, Comparison Principle, Dirichlet Problems, Neumann Problems

Mathematics Subject Classification (2010). 35J25, 35J60, 35B51, 35B99

\section{Introduction}

Let $\Omega$ be a bounded open set of $\mathbb{R}^{N}$ with Lipschitz continuous boundary, $N \geq 1$, and $m \in \mathbb{R}$. We are interested in the partial differential equation

$$
u-f=\operatorname{div}\left(u^{m} \frac{\nabla u}{|\nabla u|}\right) \quad \text { in } \Omega
$$

with $0 \leq f \in L^{\infty}(\Omega)$. Equation (1.1) corresponds to the resolvent equation of the following evolution equation:

$$
\frac{\partial u}{\partial t}=\operatorname{div}\left(u^{m} \frac{\nabla u}{|\nabla u|}\right) .
$$

When $m=0,(1.2)$ coincides with the nowadays well-known total variation flow: we refer to the monograph [10] for a detailed study of the subject and to [29] for its applications in image processing. The case $m=1$ (the so-called heat equation in transparent media) was considered in

L. Giacomelli: SBAI Department, Sapienza University of Rome, Via Scarpa 16, 00161 Roma, Italy; e-mail: lorenzo.giacomelli@sbai.uniroma1.it

S. Moll: Departament d'Anàlisi Matemàtica, Universitat de València, Spain; e-mail: j.salvador.moll@uv.es

F. Petitta: SBAI Department, Sapienza University of Rome, Via Scarpa 16, 00161 Roma, Italy; e-mail: francesco.petitta@sbai.uniroma1.it 
[7], where existence and uniqueness of entropy solutions to the Cauchy problem for both (1.1) and (1.2) were obtained. In addition, it was shown in [7] that solutions to the relativistic heat equation

$$
\frac{\partial u}{\partial t}=\varrho \operatorname{div}\left(u \frac{\nabla u}{\sqrt{u^{2}+\varrho^{2}|\nabla u|^{2}}}\right),
$$

converge to solutions of (1.2) (with $m=1$ ) as $\varrho \rightarrow+\infty$. For $m>1$, equation (1.2) is the formal limit of the relativistic porous medium equation,

$$
\frac{\partial u}{\partial t}=\varrho \operatorname{div}\left(\frac{u^{m} \nabla u}{\sqrt{u^{2}+\varrho^{2}|\nabla u|^{2}}}\right), \quad m>1,
$$

as the kinematic viscosity $\varrho$ tends to $+\infty$ (here the maximal speed of propagation has been normalized to 1). To the best of our knowledge, Eq. (1.4) was introduced in [28] while studying heat diffusion in neutral gases (precisely with $m=3 / 2$ ). Existence and uniqueness of solutions for the Cauchy problem associated to (1.4) were obtained in [5]. Some key-features of solutions, such as propagation of support, waiting time phenomena, speed of discontinuity fronts, and pattern formations, have been recently addressed by many authors $[6,18,20,22,23,15,17,16]$.

Three points of interest motivate the study of (1.2) and its resolvent equation also for $m \notin$ $\{0,1\}$.

(I) Shock formation, $m>1$. Besides pioneering contributions [12, 13] and numerical simulations $[9,19]$, the mechanism and the dynamics of shock formation for solutions to (1.4) is not yet fully understood (see in particular [23] for further insights). Since (1.2) and (1.4) formally coincide where $|\nabla u| \gg 1$, in particular at a discontinuity front, (1.2) may be seen as a prototype equation for investigating such phenomena. More generally, in flux-saturated diffusion equations such as (1.4), one expects to see strong interplays between hyperbolic and parabolic mechanisms: the scaling invariance of (1.2) with respect to $x$ should make these interplays more transparent and easier to study qualitatively.

(II) Large solutions, $m<0$. The analysis of qualitative phenomena, namely the initial propagation of support, also motivates the analysis of (1.2) in the case $m<0$. Indeed, assume that we are in the case $N=1$ and that a solution to (1.4) has a fixed support $[a, b]$ during a time interval $(0, T)$ (in particular, $u(t, \cdot)$ is continuous and equals 0 across its boundary, see [20]). Suppose that $\left.u\right|_{t=0}$ (hence $u(t)$ ) has unit total mass. Let $\varphi(t, \eta)$ be defined through

$$
\int_{a}^{\varphi(t, \eta)} u(t, x) d x=\eta, \quad \eta \in(0,1)
$$

Formally, the equation satisfied by $v(t, \eta):=1 / u(t, \varphi(t, \eta))$ is

$$
\begin{cases}\frac{\partial v}{\partial t}=\varrho\left(\frac{v^{1-m} v_{\eta}}{\sqrt{v^{4}+\varrho^{2} v_{\eta}^{2}}}\right)_{\eta} & \text { in }(0, T) \times(0,1) \\ v=+\infty & \text { on }[0, T] \times\{0,1\},\end{cases}
$$

i.e., $v$ is a "large solution" to (1.5.a). In [19], this lagrangian approach was used in the case $m=1$ to show some additional regularity properties for (1.3) (see also [17] for the use of this approach 
respect to Eq. (1.6) below). Letting $\rho \rightarrow \infty$, one is led to analyze the problem of large solutions for Equation (1.1) with $m<0$.

(III) Well-posedness. The last point of interest in (1.2) is of a more theoretical nature: (1.2) stands as a model for autonomous evolution equations in divergence form which, though of second order, have the same scaling of a first order nonlinear conservation law. As mentioned in (I), this structure may lead to simpler qualitative studies. However, at the level of well-posedness, it poses quite a few additional difficulties with respect to (1.4) and other flux-saturated diffusion equations, such as the speed-limited porous medium equation,

$$
u_{t}=\operatorname{div}\left(\frac{u \nabla u^{M-1}}{\sqrt{1+\left|\nabla u^{M-1}\right|^{2}}}\right), \quad M>1 .
$$

Indeed, while an existence and uniqueness theory is available for both (1.4) and (1.6), it is not yet for (1.2). As first step toward the elaboration of such theory, the aim of this paper is to give an appropriate notion of solutions to (1.1) and to discuss their existence and uniqueness.

We mainly concentrate on the Dirichlet problem,

$$
\begin{cases}u-f=\operatorname{div}\left(u^{m} \frac{\nabla u}{|\nabla u|}\right) & \text { in } \Omega \\ u=g & \text { on } \partial \Omega\end{cases}
$$

where $g \in L^{\infty}(\partial \Omega)$ is nonnegative. In fact, consistently with (II), for $m<0$ we assume that $f$ and $g$ (hence, as we shall see, solutions) are bounded away from zero. On the other hand, for $m>0$ a positive boundary datum $g$ does not guarantee positivity of the solution (see e.g. Example 6.1 $(v)$ for $f=0$ ) and, moreover, the case $g=0$ is interesting in view of the relation between (1.2) and (1.4) (see (I) and (II) above). Therefore, for $m>0$ we only assume nonnegativity of the data.

For all $m \in \mathbb{R}$, we introduce a notion of solutions for problem (1.7) (see Definitions 4.1 and 5.4) and we prove existence of solutions (see Theorems 4.3 and 5.6) as well as a contraction principle in $L^{1}(\Omega)$ (see Theorems 4.8 and 5.11). We also show that solutions of (1.7) have diffuse gradients, i.e., their jump set has zero $(N-1)$-dimensional Hausdorff measure (see Lemma 4.7 and 5.9), an insight which applies as well to the resolvent equations of (1.4) and (1.6) (cf. Remark 7.3).

According to our notion of solution, the Dirichlet boundary condition $u=g$ transforms into obstacle-type constraints which formally read as follows:

$$
\begin{array}{ccc}
u \leq g, \quad \text { with } \quad \frac{D u}{|D u|} \cdot \nu=1 \text { if } u<g & \text { when } m<0, \\
u \geq g, \quad \text { with } \quad \frac{D u}{|D u|} \cdot \nu=-1 \text { if } u>g & \text { when } m>0,
\end{array}
$$

where $\nu$ denotes the outward unit normal to $\partial \Omega$ (see e.g. [10] for the case $m=0$, in which $u=g$ turns into $\left.\frac{D u}{|D u|} \cdot \nu \in \operatorname{sign}(g-u)\right)$. Now, it is not surprising that in the $B V$-framework the boundary datum may not be attained. If this is the case, $(1.8)_{2}$ and (1.9) $)_{2}$ are natural compatibility conditions: seen together, they formally say that, while approaching $\partial \Omega$, either $u$ strictly decreases toward $g$ if $u>g$, or viceversa. The selection criterium given by the sign of $m$ can then be understood by a simple heuristic in one space dimension: assuming that $u$ is strictly monotone near $\partial \Omega,(1.7)$ reduces to

$$
m u^{m-1}\left|u^{\prime}\right|=u-f \quad \text { for } \mathrm{d}(x, \partial \Omega) \ll 1 .
$$


If for instance $m>0$, then (1.10) implies that $u=g$ can be attained only if $g-f \geq 0$, and otherwise $u \geq f>g$. The case $m<0$ is symmetric. Examples are given in Lemma 6.1(i).

Motivated by (II), we also provide preliminary information on existence or nonexistence of large solutions, i.e., solutions to

$$
\begin{cases}u-f=\operatorname{div}\left(u^{m} \frac{\nabla u}{|\nabla u|}\right) & \text { in } \Omega \\ u=+\infty & \text { on } \partial \Omega,\end{cases}
$$

where $f \in L^{\infty}(\Omega)$. We show in particular that, when $m<0$ and $\Omega$ is a ball, solutions are bounded independently of the boundary datum, a phenomenon which occurs also for $m=0$ (see [27], and [26] for the corresponding parabolic problem). On the other hand, for $m>1$ solutions with $g=n \in \mathbb{N}$ cannot converge to any $L_{l o c}^{1}$ function in $\Omega$, i.e. large solutions should not exist.

A similar (though simpler) approach leads to analogous results for the homogeneous Neumann problem (see Section 7):

$$
\begin{cases}u-f=\operatorname{div}\left(u^{m} \frac{\nabla u}{|\nabla u|}\right) & \text { in } \Omega \\ u^{m} \frac{\nabla u}{|\nabla u|} \cdot \nu=0 & \text { on } \partial \Omega .\end{cases}
$$

Also, our analysis of both (1.7) and (1.11) extends to more general forms of the nonlinearities (see Section 7).

The plan of the paper is the following: Section 2 contains definitions, notations, and known results (on divergence-measure fields and TBV-functions) used in the paper. Section 3 is devoted to the construction of suitable approximating solutions. Section 4 discusses well-posedness and regularity of solutions to (1.7) in the singular case, $m<0$. In Section 5, analogous results are proved for problem (1.7) in the degenerate case, $m>0$, with some technical complications since a priori bounds do not control $|D u|$ down to $u=0$. Due to that, a few new results on $T B V$-spaces are given in Section 5.1. Section 6 discusses qualitative features of solutions to (1.7), including global a priori $L^{\infty}(\Omega)$ bounds of solutions $(m<0)$, a barrier for the case $0<m<1$, and nonexistence of uniform bounds in case $m>1$. Section 7 deals with the case of homogeneous Neumann boundary conditions and to more general nonlinearities.

\section{Preliminaries}

\subsection{Notation}

We denote by $\mathcal{H}^{N-1}$ the $(N-1)$-dimensional Hausdorff measure, by $\mathcal{L}^{N}$ the $N$-dimensional Lebesgue measure, and by $\mathcal{M}(\Omega)$ the space of finite Radon measures on $\Omega$ (see [3, Def. 1.40]). The subscript ${ }_{0}$ denotes spaces of compactly supported functions. We recall that $\mathcal{M}(\Omega)$ is the dual space of $C_{0}(\Omega)$. We let $\mathcal{D}(\Omega):=C_{0}^{\infty}(\Omega), \mathcal{D}^{\prime}(\Omega)$ its dual, and

$$
\begin{aligned}
B V^{+}(\Omega) & =B V(\Omega) \cap L^{1}(\Omega ;[0,+\infty)), \\
D B V(\Omega) & =\left\{u \in B V(\Omega): \mathcal{H}^{N-1}\left(S_{u}\right)=0\right\} .
\end{aligned}
$$

We use standard notation and properties of $B V$ functions, for which we refer to [3]. For $a<b$, we define the truncating functions

$$
T_{a}^{b}(s):=\max (\min (b, s), a), \quad T_{a}(s):=T_{-a}^{a}(s), \quad T_{a}^{\infty}(s):=\max (s, a), \quad s \in \mathbb{R},
$$


and the spaces

$$
\mathcal{T}:=\left\{T_{a}^{b}: 0<a<b\right\}, \quad \mathcal{T}^{\infty}:=\left\{T_{a}^{\infty}: 0<a\right\} .
$$

For $F \in W_{l o c}^{1,1}((0,+\infty))$, let

$$
\phi_{F}(s):=\int_{1}^{s} F^{\prime}(\sigma) \sigma^{m} \mathrm{~d} \sigma, \quad s>0
$$

In particular,

$$
\phi(s):=\phi_{\operatorname{Id}}(s)=\left\{\begin{array}{ll}
\frac{1}{m+1} s^{m+1} & \text { if } m \neq-1 \\
\log s & \text { if } m=-1,
\end{array} \quad \text { so that } \quad \phi^{\prime}(s)=s^{m} .\right.
$$

\subsection{TBV-functions}

Let

$$
T B V^{+}(\Omega)=\left\{u \in L^{1}(\Omega ;[0,+\infty)): F(u) \in B V(\Omega) \forall a>0, F \in W_{a}^{1, \infty}\right\},
$$

where

$$
W_{a}^{1, \infty}=W^{1, \infty}([0,+\infty) ;[a,+\infty)), \quad a>0 .
$$

We now outline some properties of $T B V^{+}(\Omega)$ which are analogous to those of $G B V(\Omega)$, the space of integrable functions such that $T_{a}(u) \in B V(\Omega)$ for any $a \geq 0$ (see [3]). Further properties of the space $T B V^{+}$will be proved later in Section 5.1. First of all, $T B V^{+}$may be equivalently defined as

$$
T B V^{+}(\Omega)=\left\{u \in L^{1}(\Omega ;[0,+\infty)): T(u) \in B V(\Omega) \text { for all } T \in \mathcal{T}^{\infty}\right\}
$$

(see [3, Remark 4.27]). Given $u \in L^{1}(\Omega)$, the upper and lower approximate limits of $u$ at a point $x \in \Omega$ are defined respectively as

$$
\begin{aligned}
& u^{\vee}(x):=\inf \left\{t \in \mathbb{R}: \lim _{\rho \downarrow 0} \rho^{-N}\left|\{u>t\} \cap B_{\rho}(x)\right|=0\right\}, \\
& u^{\wedge}(x):=\sup \left\{t \in \mathbb{R}: \lim _{\rho \downarrow 0} \rho^{-N}\left|\{u<t\} \cap B_{\rho}(x)\right|=0\right\} .
\end{aligned}
$$

We let $S_{u}^{*}:=\left\{x \in \Omega: u^{\wedge}(x)<u^{\vee}(x)\right\}$ and

$$
D T B V^{+}(\Omega)=\left\{u \in T B V^{+}(\Omega): \mathcal{H}^{N-1}\left(S_{u}^{*}\right)=0\right\} .
$$

The set of weak approximate jump points is the subset $J_{u}^{*}$ of $S_{u}^{*}$ such that there exists a unit vector $\nu_{u}^{*}(x) \in \mathbb{R}^{N}$ such that the weak approximate limit of the restriction of $u$ to the hyperplane $H^{+}:=\left\{y \in \Omega:\left\langle y-x, \nu_{u}^{*}(x)\right\rangle>0\right\}$ is $u^{\vee}(x)$ and the weak approximate limit of the restriction of $u$ to $H^{-}:=\left\{y \in \Omega:\left\langle y-x, \nu_{u}^{*}(x)\right\rangle<0\right\}$ is $u^{\wedge}(x)$. In [3, Page 237] it is shown that for any $u \in L_{l o c}^{1}(\Omega), J_{u} \subset J_{u}^{*}$. Moreover, $u^{\vee}(x)=\max \left\{u^{+}(x), u^{-}(x)\right\}, u^{\wedge}(x)=\min \left\{u^{+}(x), u^{-}(x)\right\}$ and $\nu_{u}^{*}(x)= \pm \nu_{u}(x)$ for any $x \in J_{u}$. Furthermore, arguing as in [3, Theorem 4.34] one obtains the following result.

Lemma 2.1. For any $u \in T B V^{+}(\Omega) \cap L^{\infty}(\Omega)$,

(i) $S_{u}^{*}=\cup_{a>0} S_{T_{a}^{\infty}(u)}$ and

$$
u^{\vee}(x)=\lim _{a \rightarrow 0^{+}}\left(T_{a}^{\infty}(u)\right)^{\vee}(x), \quad u^{\wedge}(x)=\lim _{a \rightarrow 0^{+}}\left(T_{a}^{\infty}(u)\right)^{\wedge}(x) ;
$$

(ii) $S_{u}^{*}$ is countably $\mathcal{H}^{N-1}$ rectifiable and $\mathcal{H}^{N-1}\left(S_{u}^{*} \backslash J_{u}^{*}\right)=0$. 


\subsection{Divergence-measure vector-fields}

Let

$$
\begin{aligned}
& X(\Omega)=\left\{\mathbf{z} \in L^{\infty}\left(\Omega ; \mathbb{R}^{N}\right): \operatorname{div} \mathbf{z} \in L^{\infty}(\Omega)\right\}, \\
& X_{\mathcal{M}}(\Omega)=\left\{\mathbf{z} \in L^{\infty}\left(\Omega ; \mathbb{R}^{N}\right): \operatorname{div} \mathbf{z} \in \mathcal{M}(\Omega)\right\} .
\end{aligned}
$$

In [11, Theorem 1.2] (see also [10, 21]), the weak trace on $\partial \Omega$ of the normal component of $\mathbf{z} \in$ $X_{\mathcal{M}}(\Omega)$ is defined as a linear operator $[\cdot, \nu]: X_{\mathcal{M}}(\Omega) \rightarrow L^{\infty}(\partial \Omega)$ such that $\|[\mathbf{z}, \nu]\|_{L^{\infty}(\partial \Omega)} \leq$ $\|\mathbf{z}\|_{\infty}$ for all $\mathbf{z} \in X_{\mathcal{M}}(\Omega)$ and $[\mathbf{z}, \nu]$ coincides with the point-wise trace of the normal component if $\mathbf{z}$ is smooth:

$$
[\mathbf{z}, \nu](x)=\mathbf{z}(x) \cdot \nu(x) \quad \text { for all } x \in \partial \Omega \text { if } \mathbf{z} \in C^{1}\left(\bar{\Omega}, \mathbb{R}^{m}\right) .
$$

It follows from [21, Proposition 3.1] or [2, Proposition 3.4] that $\operatorname{div} \mathbf{z}$ is absolutely continuous with respect to $\mathcal{H}^{N-1}$. Therefore, given $\mathbf{z} \in X_{\mathcal{M}}(\Omega)$ and $u \in B V(\Omega) \cap L^{\infty}(\Omega)$, the functional $(\mathbf{z}, D u) \in \mathcal{D}^{\prime}(\Omega)$ given by

$$
\langle(\mathbf{z}, D u), \varphi\rangle:=-\int_{\Omega} u^{*} \varphi \mathrm{d}(\operatorname{div} \mathbf{z})-\int_{\Omega} u \mathbf{z} \nabla \varphi \mathrm{d} x
$$

is well defined, and the following holds (see [20], Lemma 5.1, Theorem 5.3, Lemma 5.4, and Lemma 5.6).

Lemma 2.2. Let $\mathbf{z} \in X_{\mathcal{M}}(\Omega)$ and $u \in B V(\Omega) \cap L^{\infty}(\Omega)$. Then the functional $(\mathbf{z}, D u) \in \mathcal{D}^{\prime}(\Omega)$ defined by (2.4) is a Radon measure which is absolutely continuous with respect to $|D u|$. Furthermore

$$
\begin{gathered}
\int_{\Omega} u^{*} \mathrm{~d}(\operatorname{div} \mathbf{z})+(\mathbf{z}, D u)(\Omega)=\int_{\partial \Omega}[\mathbf{z}, \nu] u \mathrm{~d} \mathcal{H}^{m-1} \\
\operatorname{div}(u \mathbf{z})=u^{*} \operatorname{div} \mathbf{z}+(\mathbf{z}, D u) \quad \text { as measures }
\end{gathered}
$$

and

$$
[u \mathbf{z}, \nu]=u[\mathbf{z}, \nu] \quad \mathcal{H}^{N-1} \text {-a.e. on } \partial \Omega .
$$

We denote by $\theta(\mathbf{z}, D u)$ the Radon-Nikodym derivative of $(\mathbf{z}, D u)$ with respect to $|D u|$. The following result can be found in [24, Proposition 2.7].

Lemma 2.3. Let $u \in D B V(\Omega) \cap L^{\infty}(\Omega), \mathbf{z} \in X_{\mathcal{M}}(\Omega)$ and let $\Gamma$ be a Lipschitz continuous nondecreasing function. Then

$$
\theta(\mathbf{z}, D(\Gamma(u)))=\theta(\mathbf{z}, D u) \quad|D(\Gamma \circ u)|-\text { a.e. in } \Omega .
$$

Consequently,

$$
(\mathbf{z}, D(\Gamma(u)))=\Gamma^{\prime}(u)(\mathbf{z}, D u) \quad \text { as measures. }
$$

In $[2, \S 3]$ (see also [20]), the normal traces $[\mathbf{z}, \Sigma]^{ \pm}$of a vector field $\mathbf{z} \in X_{\mathcal{M}}(\Omega)$ are defined on an oriented $C^{1}$-hypersurface $\Sigma \subset \Omega$ :

$$
[\mathbf{z}, \Sigma]^{ \pm}:=\left[\mathbf{z}, \nu_{\Omega^{ \pm}}\right]
$$


where $\Omega^{ \pm} \Subset \Omega$ are open $C^{1}$ domains such that $\Sigma \subset \partial \Omega^{ \pm}$and $\nu_{\Omega^{ \pm}}= \pm \nu_{\Sigma}$ (the definition is seen to be independent of $\Omega^{ \pm}$up to a set of zero $\mathcal{H}^{N-1}$-measure). In addition [2, Proposition 3.4], it is proved that

$$
(\operatorname{div} \mathbf{z})\left\llcorner\Sigma=\left([\mathbf{z}, \Sigma]^{+}-[\mathbf{z}, \Sigma]^{-}\right) \mathcal{H}^{N-1}\llcorner\Sigma .\right.
$$

By localization, this notion is then extended to oriented countably $\mathcal{H}^{N-1}$-rectifiable sets $\Sigma$ (these are countable union, up to a $\mathcal{H}^{N-1}$-negligible set, of oriented $C^{1}$-hypersurfaces). Using this definition, from (2.10) one immediately gets the following:

Lemma 2.4. Let $\mathbf{z} \in X_{\mathcal{M}}(\Omega)$ and let $\Sigma \subset \Omega$ be an oriented countably $\mathcal{H}^{N-1}$-rectifiable set. Then

$$
(\operatorname{div} \mathbf{z})\left\llcorner\Sigma=\left([\mathbf{z}, \Sigma]^{+}-[\mathbf{z}, \Sigma]^{-}\right) \mathcal{H}^{N-1}\llcorner\Sigma .\right.
$$

The next result is a consequence of Lemma 2.2.

Lemma 2.5. Let $u \in B V(\Omega) \cap L^{\infty}(\Omega)$ and $\mathbf{w} \in X_{\mathcal{M}}\left(\Omega, \mathbb{R}^{N}\right)$. Then

$$
\left[u \mathbf{w}, \nu_{u}\right]^{ \pm}=u^{ \pm}\left[\mathbf{w}, \nu_{u}\right]^{ \pm} \quad \mathcal{H}^{N-1} \text {-a.e. on } J_{u}
$$

Proof. By (2.6), the vector field $\mathbf{z}:=u \mathbf{w}$ belongs to $X_{\mathcal{M}}(\Omega)$. As shown in [3, Theorem 3.78], $J_{u}$ is a countably $\mathcal{H}^{N-1}$-rectifiable set oriented by the direction of $\nu_{u}$. Having in mind the way in which traces of $\mathbf{w}$ are defined over rectifiable sets, it suffices to prove that for any $\Omega^{\prime} \Subset \Omega$ open with a $C^{1}$ boundary, then

$$
\left[\mathbf{z}, \nu_{\Omega^{\prime}}\right]^{ \pm}=u^{ \pm}\left[\mathbf{w}, \nu_{\Omega^{\prime}}\right]^{ \pm} \quad \mathcal{H}^{N-1} \text {-a.e. on } \partial \Omega^{\prime}
$$

which follows directly from Lemma 2.2.

We conclude with two properties of the pairing (2.4) for bounded $D B V$-functions.

Lemma 2.6. Let $\mathbf{z} \in X_{\mathcal{M}}(\Omega)$ and let $u, v \in D B V(\Omega) \cap L^{\infty}(\Omega)$. Then

$$
\begin{gathered}
(u \mathbf{z}, D v)=u(\mathbf{z}, D v) \text { as measures, } \\
(\mathbf{z}, D(u v))=u(\mathbf{z}, D v)+v(\mathbf{z}, D u)=(u \mathbf{z}, D v)+(v \mathbf{z}, D u) .
\end{gathered}
$$

Proof. The proof of (2.12) follows line by line the one in [25, Proposition 2.3] which is based on Lemma 2.3 above. A repeated application of Lemma 2.2 gives

$$
\begin{aligned}
(\mathbf{z}, D(u v)) & =-u v \operatorname{div} \mathbf{z}+\operatorname{div}(u v \mathbf{z}) \\
& =-u(\operatorname{div}(v \mathbf{z})-(\mathbf{z}, D v))+u \operatorname{div}(v \mathbf{z})+(v \mathbf{z}, D u) \\
& =u(\mathbf{z}, D v)+(v \mathbf{z}, D u)
\end{aligned}
$$

and (2.13) follows from (2.12). 


\section{Approximating problems}

We let

$$
|\eta|_{\varepsilon}:=\sqrt{|\eta|^{2}+\varepsilon^{2}}
$$

and we note that

$$
\frac{|\eta|^{2}}{|\eta|_{\varepsilon}}=\frac{|\eta|_{\varepsilon}^{2}-\varepsilon^{2}}{|\eta|_{\varepsilon}} \geq|\eta|-\varepsilon
$$

For $\varepsilon \in(0,1)$ we consider the following approximating problems:

$$
\begin{cases}u-f=\operatorname{div}\left((\varepsilon+|u|)^{m} \frac{\nabla u}{|\nabla u|_{\varepsilon}}+\varepsilon \nabla u\right) & \text { in } \Omega \\ u=g & \text { on } \partial \Omega .\end{cases}
$$

In this section, using standard monotonicity arguments (see for instance [14] and [30]), we prove the following result.

Lemma 3.1. For any $m \in \mathbb{R}$, any $f \in L^{\infty}(\Omega)$, and any $g \in L^{\infty}(\partial \Omega)$, there exists a solution $u_{\varepsilon} \in H^{1}(\Omega) \cap L^{\infty}(\Omega)$ of (3.2) with data $(f, g)$ in the sense that

$$
\int_{\Omega}\left(u_{\varepsilon}-f\right) \varphi=-\int_{\Omega}\left(\left(\varepsilon+\left|u_{\varepsilon}\right|\right)^{m} \frac{\nabla u_{\varepsilon}}{\left|\nabla u_{\varepsilon}\right|_{\varepsilon}}+\varepsilon \nabla u_{\varepsilon}\right) \cdot \nabla \varphi \quad \text { for all } \varphi \in H_{0}^{1}(\Omega)
$$

and $u_{\varepsilon}=g$ on $\partial \Omega$. Furthermore,

$$
\left\|u_{\varepsilon}\right\|_{L^{\infty}(\Omega)} \leq \max \left\{\|f\|_{L^{\infty}(\Omega)},\|g\|_{L^{\infty}(\partial \Omega)}\right\}
$$

and $u_{\varepsilon} \geq 0$ if $f \geq 0$ and $g \geq 0$.

Proof. Fix $\delta>0$ and consider the following auxiliary problems:

$$
\begin{cases}u-f=\operatorname{div}\left(\left(\varepsilon+T_{1 / \delta}(|u|)\right)^{m} \frac{\nabla u}{|\nabla u|_{\varepsilon}}+\varepsilon \nabla u\right) & \text { in } \Omega \\ u=g & \text { on } \partial \Omega .\end{cases}
$$

Fix $\tilde{g} \in H^{1}(\Omega)$ such that $\tilde{g}=g$ on $\partial \Omega$, let $w=u-\tilde{g}$, and let

$$
A_{0}(x, w)=w+\tilde{g}, \quad A_{1}(x, w, \boldsymbol{\xi})=\left(\varepsilon+T_{1 / \delta}(|w+\tilde{g}|)\right)^{m} \frac{\boldsymbol{\xi}+\nabla \tilde{g}}{|\boldsymbol{\xi}+\nabla \tilde{g}|_{\varepsilon}}+\varepsilon(\boldsymbol{\xi}+\nabla \tilde{g}) .
$$

Then (3.5) is equivalent to solving

$$
A_{0}(x, w)-\operatorname{div}\left(A_{1}(x, w, \nabla w)\right)=f, \quad w=0 \text { on } \partial \Omega .
$$

We note that

$$
0 \leq\left(\varepsilon+T_{1 / \delta}(|w+\tilde{g}|)\right)^{m} \leq C
$$

for some $C>0$ (depending on $\varepsilon, \delta$, and $m$ ). Existence of solutions follows from, e.g., [14, Corollary 1] with $p=2$ in the space $H_{0}^{1}(\Omega)$. For its applicability, we need to check:

- boundedness of $\left|A_{0}\right|$ and $\left|A_{1}\right|$, which follows from

$$
\left|A_{0}(x, w)\right|+\left|A_{1}(x, w, \boldsymbol{\xi})\right| \stackrel{(3.6)}{\leq}|w|+\varepsilon|\boldsymbol{\xi}|+\underbrace{C+|\tilde{g}|+\varepsilon|\nabla \tilde{g}|}_{\in L^{2}(\Omega)}
$$


- monotonicity of $A_{1}$, in form of

$$
\left(A_{1}\left(x, w, \boldsymbol{\xi}_{1}\right)-A_{1}\left(x, w, \boldsymbol{\xi}_{2}\right)\right) \cdot\left(\boldsymbol{\xi}_{1}-\boldsymbol{\xi}_{2}\right)>0 \quad \text { for all } \boldsymbol{\xi}_{1} \neq \boldsymbol{\xi}_{2},
$$

which follows from the convexity of the associated Lagrangian,

$$
f(x, w, \boldsymbol{\xi})=\left(\varepsilon+T_{1 / \delta}(|w+\tilde{g}|)\right)^{m}|\boldsymbol{\xi}+\nabla \tilde{g}|_{\varepsilon}+\frac{\varepsilon}{2}|\boldsymbol{\xi}+\nabla \tilde{g}|^{2} \quad\left(\nabla_{\boldsymbol{\xi}} f=A_{1}\right)
$$

- coercivity, which follows from

$$
A_{1}(x, w, \boldsymbol{\xi}) \cdot \boldsymbol{\xi} \stackrel{(3.6)}{\geq} \frac{\varepsilon}{2}|\boldsymbol{\xi}|^{2}-\underbrace{\left(C|\nabla \tilde{g}|+\frac{\varepsilon}{2}|\nabla \tilde{g}|^{2}\right)}_{\in L^{1}(\Omega)} .
$$

Uniqueness easily follows by monotonicity. Therefore (3.5) has a unique solution, $u_{\varepsilon, \delta}$. Let $k:=$ $\max \left\{\|f\|_{L^{\infty}(\Omega)},\|g\|_{L^{\infty}(\partial \Omega)}\right\}$, and use $\left(u_{\varepsilon, \delta}-k\right)_{+}:=\max \left(u_{\varepsilon, \delta}-k, 0\right)$ as test function in (3.3). We obtain

$$
\int_{\Omega}\left(u_{\varepsilon, \delta}-k\right)_{+}\left(u_{\varepsilon, \delta}-f\right) \leq 0,
$$

hence $u_{\varepsilon, \delta} \leq k$. Choosing $\delta<1 / k$, we have $T_{1 / \delta}\left(\left|u_{\varepsilon, \delta}\right|\right)=\left|u_{\varepsilon, \delta}\right|$, hence $u_{\varepsilon}:=u_{\varepsilon, \delta}$ is a solution to (3.2). Provided $g \geq 0$, choosing $u_{-}:=\max \{0,-u\}$ as test function in (3.3) we obtain

$$
\int_{\Omega}\left(u_{\varepsilon}\right)_{-}\left(u_{\varepsilon}-f\right) \geq 0
$$

hence $u_{\varepsilon} \geq 0$ if both $f \geq 0$ and $g \geq 0$.

\section{The singular case}

In this section we study (1.7) in the singular case, $m<0$. We assume:

$$
\begin{gathered}
f \in L^{\infty}(\Omega), \quad f \geq 0, \\
g \in L^{\infty}(\partial \Omega), g \geq G_{0}>0 .
\end{gathered}
$$

Our definition of solution is the following.

Definition 4.1. Assume $m<0$, (4.1), and (4.2). A function $u: \Omega \mapsto[0,+\infty)$ is a solution to problem (1.7) with data $(f, g)$ if $u \in B V(\Omega) \cap L^{\infty}(\Omega), 1 / u \in L^{\infty}(\Omega)$, and there exists a gradient-director field $\mathbf{w} \in X_{\mathcal{M}}(\Omega)$ such that $\|\mathbf{w}\|_{\infty} \leq 1$ and $\mathbf{z}:=u^{m} \mathbf{w}$ satisfies

$$
\begin{gathered}
|D \phi(u)| \leq(\mathbf{z}, D u) \quad \text { as measures in } \Omega, \\
u-f=\operatorname{div} \mathbf{z} \quad \text { in } \mathcal{D}^{\prime}(\Omega)
\end{gathered}
$$

and

$$
\begin{gathered}
u \leq g \quad \mathcal{H}^{N-1} \text {-a.e. on } \partial \Omega, \\
{[\mathbf{z}, \nu]=u^{m} \text { if } u<g \quad \mathcal{H}^{N-1} \text {-a.e. on } \partial \Omega .}
\end{gathered}
$$


Remark 4.2. Since $s \mapsto s^{m}$ is locally Lipschitz in $(0,+\infty)$,

$$
\left.\begin{array}{l}
u \in B V(\Omega) \cap L^{\infty}(\Omega ;[0,+\infty)) \\
1 / u \in L^{\infty}(\Omega)
\end{array}\right\} \Rightarrow u^{m} \in B V(\Omega) \cap L^{\infty}(\Omega) .
$$

In addition, by (4.1) and (4.4), $\operatorname{div} \mathbf{z} \in L^{\infty}(\Omega)$; hence $\mathbf{z} \in X(\Omega)$.

The main result of this section is the following.

Theorem 4.3. Assume $m<0$, (4.1), and (4.2). Then there exists a unique solution $u$ of (1.7) with data $(f, g)$ in the sense of Definition 4.1. In addition, $u \in D B V(\Omega)$ and

$$
|D \phi(u)|=(\mathbf{z}, D u) \quad \text { and } \quad(\mathbf{w}, u)=|D u| \quad \text { as measures in } \Omega .
$$

\subsection{Existence}

The proof of the existence part of Theorem 4.3 follows from Lemmas 4.4-4.7 below.

Lemma 4.4 (A priori lower bound). Assume $m<0$, (4.1), and (4.2). Positive constants $\alpha$ and $\varepsilon_{0}$, depending only on $\Omega$ and $G_{0}$, exist such that for any $\varepsilon<\varepsilon_{0}$ the solution $u_{\varepsilon}$ of (3.2) with data $(f, g)$ satisfies

$$
u_{\varepsilon} \geq \alpha>0
$$

Proof. Let $R>0$ be such that $\Omega \subset B(0 ; R)$. We choose

$$
\begin{gathered}
0<\alpha<\min \left\{\left(\frac{1}{2^{3-m}\left(1+R^{2}\right)^{3 / 2}}\right)^{\frac{1}{1-m}}, G_{0}\right\}, \\
0<\varepsilon_{0}<\min \left\{\frac{G_{0}-\alpha}{R^{2}}, \frac{\alpha}{2|m| R^{2}\left(1+R^{2}\right)}, \frac{2 \alpha}{2+R^{2}}\right\} .
\end{gathered}
$$

We claim that $v_{\varepsilon}(x):=\varepsilon \frac{|x|^{2}}{2}+\alpha$ is a subsolution to (3.2) for any $0<\varepsilon<\varepsilon_{0}$. On one hand,

$$
\begin{aligned}
\operatorname{div} & \left(\left(\varepsilon+v_{\varepsilon}\right)^{m} \frac{\nabla v_{\varepsilon}}{\left|\nabla v_{\varepsilon}\right|_{\varepsilon}}+\varepsilon \nabla v_{\varepsilon}\right) \\
& =\left(\varepsilon+v_{\varepsilon}\right)^{m-1} \frac{m \varepsilon|x|^{2}}{\sqrt{1+|x|^{2}}}+\left(\varepsilon+v_{\varepsilon}\right)^{m}\left(\frac{1}{\left(1+|x|^{2}\right)^{3 / 2}}+\frac{N-1}{\sqrt{1+|x|^{2}}}\right)+\varepsilon^{2} N \\
> & \left(\varepsilon+v_{\varepsilon}\right)^{m-1}\left(\frac{m \varepsilon|x|^{2}}{\sqrt{1+|x|^{2}}}+\frac{\alpha}{\left(1+|x|^{2}\right)^{3 / 2}}\right) \quad\left(\text { since } v_{\varepsilon}>\alpha\right) \\
> & \frac{\left(\varepsilon+v_{\varepsilon}\right)^{m-1}}{\sqrt{1+R^{2}}}\left(m \varepsilon R^{2}+\frac{\alpha}{1+R^{2}}\right) \quad\left(\text { since }[0,+\infty) \ni s \mapsto \frac{s}{\sqrt{1+s}}\right. \text { increases) } \\
> & \frac{\left(\varepsilon+v_{\varepsilon}\right)^{m-1} \alpha}{2\left(1+R^{2}\right)^{3 / 2}} \quad\left(\text { since } \varepsilon<\frac{\alpha}{2|m| R^{2}\left(1+R^{2}\right)}\right) .
\end{aligned}
$$

On the other hand,

$$
\left.v_{\varepsilon}-f<\varepsilon+v_{\varepsilon} \leq \varepsilon+\alpha+\varepsilon \frac{R^{2}}{2}<2 \alpha \quad \text { (since } v_{\varepsilon}<\varepsilon+\frac{R^{2}}{2} \text { and } \varepsilon<\frac{2 \alpha}{2+R^{2}}\right) .
$$


Because of (4.9) and (4.10),

$$
v_{\varepsilon}-f<\operatorname{div}\left(\left(\varepsilon+v_{\varepsilon}\right)^{m} \frac{\nabla v_{\varepsilon}}{\left|\nabla v_{\varepsilon}\right|_{\varepsilon}}+\varepsilon \nabla v_{\varepsilon}\right)
$$

is implied by

$$
(2 \alpha)^{2-m}<\frac{\alpha}{2\left(1+R^{2}\right)^{3 / 2}},
$$

which is true by (4.7). The two additional constraints in (4.7) and (4.8) guarantee that $v_{\varepsilon} \leq g$ on $\partial \Omega$. This, together with (4.11), implies that $v_{\varepsilon} \leq u_{\varepsilon}$ in $\Omega$ : the argument is analogous, though simpler, to the one used in the proof of Theorem 4.8 below, and therefore we omit it.

Lemma 4.5 (Passage to the limit). Assume $m<0$, (4.1), and (4.2). Then there exists $\alpha>0$ and a pair $(u, \mathbf{w}) \in B V(\Omega) \times L^{\infty}\left(\Omega ; \mathbb{R}^{N}\right)$ such that $\|\mathbf{w}\|_{\infty} \leq 1$,

$$
0<\alpha \leq u \leq \max \left\{\|f\|_{L^{\infty}(\Omega)},\|g\|_{L^{\infty}(\partial \Omega)}\right\}
$$

$\mathbf{z}=u^{m} \mathbf{w}$ verifies

$$
u-f=\operatorname{div} \mathbf{z} \quad \text { in } \mathcal{D}^{\prime}(\Omega)
$$

and

$$
\begin{aligned}
\left|D \phi_{F}(u)\right| & \leq(\mathbf{z}, D F(u)) \quad \text { as measures } \\
\left|\phi_{F}(u)-\phi_{F}(g)\right| & \leq(F(g)-F(u))[\mathbf{z}, \nu] \quad \mathcal{H}^{N-1} \text {-a.e. on } \partial \Omega
\end{aligned}
$$

for any nondecreasing $F \in W_{l o c}^{1, \infty}((0,+\infty))$, where $\phi_{F}$ is defined by (2.1). In particular,

$$
\begin{aligned}
|D \phi(u)| & \leq(\mathbf{z}, D u) \quad \text { as measures } \\
|\phi(u)-\phi(g)| & \leq(g-u)[\mathbf{z}, \nu] \quad \mathcal{H}^{N-1} \text {-a.e. on } \partial \Omega .
\end{aligned}
$$

Proof. Up to (4.13), the proof is rather standard. Let $u_{\varepsilon}$ be as in Lemma 3.1. Lemma 4.4 and (3.4) guarantee that there exists $\alpha>0$ such that

$$
0<\alpha \leq u_{\varepsilon} \leq \max \left\{\|f\|_{L^{\infty}(\Omega)},\|g\|_{L^{\infty}(\partial \Omega)}\right\} .
$$

We define

$$
\mathbf{w}_{\varepsilon}:=\frac{\nabla u_{\varepsilon}}{\left|\nabla u_{\varepsilon}\right|_{\varepsilon}}, \quad \tilde{\mathbf{z}}_{\varepsilon}:=\left(\varepsilon+u_{\varepsilon}\right)^{m} \mathbf{w}_{\varepsilon}, \quad \mathbf{z}_{\varepsilon}:=\tilde{\mathbf{z}}_{\varepsilon}+\varepsilon \nabla u_{\varepsilon} .
$$

Let $\tilde{g} \in H^{1}\left(\Omega ;\left[G_{0},\|g\|_{L^{\infty}(\partial \Omega)}\right]\right)$ such that $\tilde{g}=g$ on $\partial \Omega$. We agree that $\int f \mathrm{~d} \mu=\int_{\Omega} f \mathrm{~d} \mu$ and that $\int f=\int f \mathrm{~d} x$. Choosing $\varphi=\left(u_{\varepsilon}-\tilde{g}\right)$ in (3.3), we obtain

$$
\begin{aligned}
\int\left(u_{\varepsilon}-\tilde{g}\right)\left(u_{\varepsilon}-f\right) & =-\int\left(\varepsilon+u_{\varepsilon}\right)^{m} \frac{\left|\nabla u_{\varepsilon}\right|^{2}}{\left|\nabla u_{\varepsilon}\right|_{\varepsilon}}-\varepsilon \int\left|\nabla u_{\varepsilon}\right|^{2}+\int \mathbf{z}_{\varepsilon} \cdot \nabla \tilde{g} \\
& \stackrel{(3.1)}{\leq}-\int\left(\varepsilon+u_{\varepsilon}\right)^{m}\left(\left|\nabla u_{\varepsilon}\right|-\varepsilon\right)-\varepsilon \int\left|\nabla u_{\varepsilon}\right|^{2}+\int \mathbf{z}_{\varepsilon} \cdot \nabla \tilde{g} .
\end{aligned}
$$

In what follows, $C \geq 1$ denotes a generic constant independent of $\varepsilon \in(0,1)$. In view of (4.18) we have

$$
C^{-1} \leq\left(\varepsilon+u_{\varepsilon}\right)^{m} \leq C
$$


and

$$
\left|\tilde{\mathbf{z}}_{\varepsilon}\right| \stackrel{(4.19)}{\leq}\left(\varepsilon+u_{\varepsilon}\right)^{m} \stackrel{(4.20)}{\leq} C
$$

Hence

$$
\int u_{\varepsilon}^{2}+\int\left|\nabla u_{\varepsilon}\right|+\varepsilon \int\left|\nabla u_{\varepsilon}\right|^{2} \leq C \int\left(\varepsilon+\left|f\left(u_{\varepsilon}-\tilde{g}\right)\right|+u_{\varepsilon} \tilde{g}+|\nabla \tilde{g}|+\varepsilon\left|\nabla u_{\varepsilon} \cdot \nabla \tilde{g}\right|\right)
$$

and by Hölder and Cauchy-Schwarz inequalities

$$
\int u_{\varepsilon}^{2}+\int\left|\nabla u_{\varepsilon}\right|+\varepsilon \int\left|\nabla u_{\varepsilon}\right|^{2} \leq C\left(1+\int f^{2}+\int \tilde{g}^{2}+\int|\nabla \tilde{g}|^{2}\right) .
$$

By (4.18) and (4.21), along subsequences (not relabeled) we obtain the existence of $u \in B V(\Omega) \cap$ $L^{\infty}(\Omega)$ and $\mathbf{w} \in L^{\infty}\left(\Omega ; \mathbb{R}^{N}\right)$ such that

$$
\begin{aligned}
u_{\varepsilon} \stackrel{*}{\rightarrow} u & \text { in } B V(\Omega) \text { and in } L^{\infty}(\Omega) \\
u_{\varepsilon} \rightarrow u & \mathcal{L}^{N} \text {-a.e. and in } L^{p}(\Omega) \text { for all } p<+\infty \\
\varepsilon \nabla u_{\varepsilon} \rightarrow 0 & \text { strongly in } L^{2}\left(\Omega ; \mathbb{R}^{N}\right) \\
\mathbf{w}_{\varepsilon} \stackrel{*}{\rightarrow} \mathbf{w} & \text { in } L^{\infty}\left(\Omega ; \mathbb{R}^{N}\right) \\
\tilde{\mathbf{z}}_{\varepsilon} \stackrel{*}{\rightarrow} \mathbf{z}=u^{m} \mathbf{w} & \text { in } L^{\infty}\left(\Omega ; \mathbb{R}^{N}\right) .
\end{aligned}
$$

In addititon, (4.12) holds. The limits in (4.23) and (4.25) combine into

$$
\mathbf{z}_{\varepsilon} \rightarrow \mathbf{z} \quad \text { in } L^{2}\left(\Omega ; \mathbb{R}^{N}\right)
$$

The bound in (4.12) follows from (4.18) and the identity in (4.13) follows from (3.2), (4.22), and (4.26).

Let $F \in W_{l o c}^{1, \infty}((0,+\infty))$ be nondecreasing and $\varphi \in C^{\infty}(\Omega)$ be nonnegative. Testing (3.2) by $\varphi\left(F\left(u_{\varepsilon}\right)-F(\tilde{g})\right)$, after an integration by parts we obtain

$$
\begin{gathered}
\int \varphi F^{\prime}\left(u_{\varepsilon}\right) \mathbf{z}_{\varepsilon} \cdot \nabla u_{\varepsilon} \\
=\int \varphi \mathbf{z}_{\varepsilon} \cdot \nabla F(\tilde{g})-\int \varphi\left(F\left(u_{\varepsilon}\right)-F(\tilde{g})\right)\left(u_{\varepsilon}-f\right)-\int\left(F\left(u_{\varepsilon}\right)-F(\tilde{g})\right) \mathbf{z}_{\varepsilon} \cdot \nabla \varphi .
\end{gathered}
$$

On the right-hand side we pass to the limit as $\varepsilon \rightarrow 0$ using (4.18), (4.22) and (4.26):

$$
\begin{aligned}
& \lim _{\varepsilon \rightarrow 0} \varphi F^{\prime}\left(u_{\varepsilon}\right) \mathbf{z}_{\varepsilon} \cdot \nabla u_{\varepsilon} \\
& =\int \varphi \mathbf{z} \cdot \nabla F(\tilde{g})-\int \varphi(F(u)-F(\tilde{g}))(u-f)-\int(F(u)-F(\tilde{g})) \mathbf{z} \cdot \nabla \varphi \\
& \stackrel{(4.13)}{=} \int \varphi \mathbf{z} \cdot \nabla F(\tilde{g})-\int \varphi(F(u)-F(\tilde{g})) \operatorname{div} \mathbf{z}-\int(F(u)-F(\tilde{g})) \mathbf{z} \cdot \nabla \varphi .
\end{aligned}
$$

Note that, by (4.12), $F(u) \in B V(\Omega)$. Integrating by parts on the right-hand side of (4.27) and using Lemma 2.2, we see that

$$
\lim _{\varepsilon \rightarrow 0} \int \varphi F^{\prime}\left(u_{\varepsilon}\right) \mathbf{z}_{\varepsilon} \cdot \nabla u_{\varepsilon}=\int \varphi \mathrm{d}(\mathbf{z}, D F(u))-\int_{\partial \Omega} \varphi(F(u)-F(g))[\mathbf{z}, \nu] \mathrm{d} \mathcal{H}^{N-1} .
$$


Since

$$
\begin{aligned}
& \int \varphi F^{\prime}\left(u_{\varepsilon}\right) \mathbf{z}_{\varepsilon} \cdot \nabla u_{\varepsilon}=\int \varphi F^{\prime}\left(u_{\varepsilon}\right)\left(\left(\varepsilon+u_{\varepsilon}\right)^{m} \frac{\left|\nabla u_{\varepsilon}\right|^{2}}{\left|\nabla u_{\varepsilon}\right|_{\varepsilon}}+\varepsilon\left|\nabla u_{\varepsilon}\right|^{2}\right) \\
& \stackrel{\text { (3.1) }}{\geq} o_{\varepsilon}(1)+\int \varphi F^{\prime}\left(u_{\varepsilon}\right) u_{\varepsilon}^{m}\left|\nabla u_{\varepsilon}\right| \text { as } \varepsilon \rightarrow 0,
\end{aligned}
$$

from (4.28) and (2.1) we derive

$$
\lim _{\varepsilon \rightarrow 0} \int \varphi\left|\nabla \phi_{F}\left(u_{\varepsilon}\right)\right| \leq \int \varphi \mathrm{d}(\mathbf{z}, D F(u))-\int_{\partial \Omega} \varphi(F(u)-F(g))[\mathbf{z}, \nu] \mathrm{d} \mathcal{H}^{N-1} .
$$

Hence, by lower semi-continuity ([8, Theorem 1])

$$
\begin{aligned}
& \int \varphi \mathrm{d}\left|D \phi_{F}(u)\right|+\int_{\partial \Omega} \varphi\left|\phi_{F}(u)-\phi_{F}(g)\right| \mathrm{d} \mathcal{H}^{N-1} \\
& \leq \int \varphi \mathrm{d}(\mathbf{z}, D F(u))-\int_{\partial \Omega} \varphi(F(u)-F(g))[\mathbf{z}, \nu] \mathrm{d} \mathcal{H}^{N-1},
\end{aligned}
$$

which yields (4.14) and (4.15) by the arbitrariness of $\varphi$.

Lemma 4.6 (Trace inequality). Let $u, \mathbf{w}$ and $\mathbf{z}$ be as in Lemma 4.5. Then $\mathbf{w} \in X_{\mathcal{M}}(\Omega)$,

$$
\begin{gathered}
{[\mathbf{z}, \nu]=u^{m}[\mathbf{w}, \nu] \quad \mathcal{H}^{N-1} \text {-a.e. on } \partial \Omega,} \\
u \leq g \quad \mathcal{H}^{N-1} \text {-a.e. on } \partial \Omega,
\end{gathered}
$$

and

$$
[\mathbf{w}, \nu]=1 \quad \mathcal{H}^{N-1} \text {-a.e. on } \partial \Omega \cap\{u<g\} .
$$

Proof. Arguing as in Remark 4.2, we see that both $u^{m}$ and $u^{-m}$ belong to $\in B V(\Omega) \cap L^{\infty}(\Omega)$. Hence, using (2.6), we have

$$
\operatorname{div} \mathbf{w}=\operatorname{div}\left(u^{-m} \mathbf{z}\right)=\left(u^{-m}\right)^{*} \operatorname{div} \mathbf{z}+\left(\mathbf{z}, D u^{-m}\right),
$$

so that $\mathbf{w} \in X_{\mathcal{M}}(\Omega)$ and (4.29) follows from (2.7) (applied with $\mathbf{z}$ replaced by $\mathbf{w}$ ).

By (4.17), we have

$$
|\phi(u)-\phi(g)| \leq(g-u)[\mathbf{z}, \nu] \stackrel{(4.29)}{\leq} u^{m}|g-u| \quad \mathcal{H}^{N-1} \text {-a.e. on } \partial \Omega .
$$

In particular, $(g-u)[\mathbf{z}, \nu] \geq 0$. Since $\phi^{\prime}(s)=s^{m}$ is strictly decreasing, $|\phi(u)-\phi(g)|>\mid u-$ $g \mid \min \left\{u^{m}, g^{m}\right\}$. Therefore, (4.32) implies that

$$
\min \left\{u^{m}, g^{m}\right\}|g-u|<(g-u)[\mathbf{z}, \nu] \leq u^{m}|g-u| \quad \mathcal{H}^{N-1} \text {-a.e. on } \partial \Omega
$$

whenever $g \neq u$. Hence $g^{m}<u^{m}$ if $g \neq u$, which means that (4.30) holds.

Let $p>0$. Choosing $F(s)=-\frac{s^{m p}}{p}$ in (4.15), and, therefore, $\phi_{F}(s)=\frac{1-s^{m(p+1)}}{p+1}$, then

$$
\left|\frac{u^{m(p+1)}-g^{m(p+1)}}{p+1}\right| \leq-\frac{g^{m p}-u^{m p}}{p}[\mathbf{z}, \nu] \mathcal{H}^{n-1} \text {-a.e. on } \partial \Omega \text {. }
$$


Using the sign properties in (4.30), we obtain

$$
\begin{aligned}
& \frac{p}{p+1}\left(u^{m(p+1)}-g^{m(p+1)}\right) \stackrel{(4.33)}{\leq}\left(u^{m p}-g^{m p}\right)[\mathbf{z}, \nu] \\
&=\left(u^{m(p+1)}-u^{m} g^{m p}\right) \frac{[\mathbf{z}, \nu]}{u^{m}} \\
& \leq\left(u^{m(p+1)}-g^{m(p+1)}\right) \frac{[\mathbf{z}, \nu]}{u^{m}}
\end{aligned}
$$

$\mathcal{H}^{N-1}$-a.e. on $\partial \Omega$. Therefore

$$
\frac{p}{p+1} \leq \frac{[\mathbf{z}, \nu]}{u^{m}} \stackrel{(4.29)}{=}[\mathbf{w}, \nu] \mathcal{H}^{N-1} \text {-a.e. on } \partial \Omega \cap\{g>u\} .
$$

Passing to the limit as $p \rightarrow+\infty$, (4.34) implies that $[\mathbf{w}, \nu]=1$ when $u<g$, and (4.31) follows.

The existence part of Theorem 4.3 is an immediate consequence of the previous Lemmas.

Proof of Theorem 4.3 (Existence). The pair $(u, \mathbf{w})$ in Lemma 4.5 has the desired regularity and satisfies (4.3) and (4.4) (see (4.16) and (4.13)). The boundary constraints (4.5) follow from Lemma 4.6.

\subsection{Regularity}

We now prove the regularity part of Theorem 4.3.

Lemma 4.7 (Regularity of $u$ and identification of $(\mathbf{w}, D u)$ ). Let $u$ be a solution to (1.7) in the sense of Definition 4.1. Then $u \in D B V(\Omega)$ and (4.6) holds true.

Proof. Arguing as in Remark 4.2, we see that $u^{m} \in B V(\Omega) \cap L^{\infty}(\Omega)$. By [3, Proposition 3.69], $J_{u^{m}}=J_{\phi(u)}=J_{u}$ and $\nu_{u^{m}}=-\nu_{u}$. Since $\mathbf{z} \in X(\Omega)$, Lemma 2.4 implies that

$$
0=(\operatorname{div} \mathbf{z})\left\llcorner J_{u}=\left(\left[\mathbf{z}, \nu_{u}\right]^{+}-\left[\mathbf{z}, \nu_{u}\right]^{-}\right) \mathcal{H}^{N-1}\left\llcorner J_{u}\right.\right.
$$

hence

$$
\Psi:=\left[\mathbf{z}, \nu_{u}\right]^{+}=\left[\mathbf{z}, \nu_{u}\right]^{-} \quad \mathcal{H}^{N-1} \text {-a.e. on } J_{u} .
$$

Applying Lemma 2.5 with $u=u^{m}$ yields

$$
\left|\left[\mathbf{z}, \nu_{u^{m}}\right]^{ \pm}\right|=\left|\left(u^{m}\right)^{ \pm}\left[\mathbf{w}, \nu_{u}\right]^{\mp}\right| \leq\left(u^{m}\right)^{ \pm} \quad \mathcal{H}^{N-1} \text {-a.e. on } J_{u} .
$$

Therefore

$$
|\Psi| \stackrel{(4.35),(4.36)}{\leq} \min \left\{\left(u^{m}\right)^{+},\left(u^{m}\right)^{-}\right\}=\min \left\{\phi^{\prime}\left(u^{+}\right), \phi^{\prime}\left(u^{-}\right)\right\}
$$

$\mathcal{H}^{N-1}$-a.e. on $J_{u}$. On the other hand,

$$
|D \phi(u)| \stackrel{(4.3)}{\leq}(\mathbf{z}, D u) \stackrel{(2.6)}{=}-u^{*} \operatorname{div} \mathbf{z}+\operatorname{div}(u \mathbf{z}) \quad \text { as measures. }
$$

Using again that $\mathbf{z} \in X(\Omega)$, this yields

$$
\left|D^{j} \phi(u)\right| \leq(\operatorname{div}(u \mathbf{z}))\left\llcorner_{J_{u}} .\right.
$$


Applying once more Lemmas 2.4 and 2.5, we obtain from (4.38):

$$
\begin{aligned}
& \left|\phi\left(u^{+}\right)-\phi\left(u^{-}\right)\right| \mathcal{H}^{N-1}\left\llcornerJ _ { u } \stackrel { ( 4 . 3 8 ) } { \leq } \operatorname { d i v } ( u \mathbf { z } ) \mathcal { H } ^ { N - 1 } \left\llcorner J_{u}\right.\right. \\
& =\left(\left[u \mathbf{z}, \nu_{u}\right]^{+}-\left[u \mathbf{z}, \nu_{u}\right]^{-}\right) \mathcal{H}^{N-1}\left\llcorner J_{u}\right. \\
& =\left(u^{+}\left[\mathbf{z}, \nu_{u}\right]^{+}-u^{-}\left[\mathbf{z}, \nu_{u}\right]^{-}\right) \mathcal{H}^{N-1}\left\llcorner J_{u}\right. \\
& \stackrel{(4.35)}{=}\left(u^{+}-u^{-}\right) \Psi \mathcal{H}^{N-1}\left\llcorner J_{u}\right. \\
& \stackrel{\text { (4.37) }}{\leq}\left|u^{+}-u^{-}\right| \min \left\{\phi^{\prime}\left(u^{+}\right), \phi^{\prime}\left(u^{-}\right)\right\} \mathcal{H}^{N-1} \mathbf{L} J_{u} \text {. }
\end{aligned}
$$

Since $\phi^{\prime}(s)=s^{m}$ is strictly monotone, we conclude that $\mathcal{H}^{N-1}\left(J_{u}\right)=0$, hence $u \in D B V(\Omega)$. Consequently, by the chain rule for $B V$-functions,

$$
u^{m}|\tilde{D} u|=|D \phi(u)| \stackrel{(4.3)}{\leq}(\mathbf{z}, D u) \stackrel{(2.12)}{=} u^{m}(\mathbf{w}, D u) \leq u^{m}|\tilde{D} u|
$$

as measures (recall that $\tilde{D} u$ denotes the diffuse part of the gradient of $u$ ). Therefore $(\mathbf{z}, D u)=$ $|D \phi(u)|$ and $(\mathbf{w}, D u)=|D u|$.

\subsection{Comparison and Uniqueness}

We have the following contraction principle for solutions to (1.7).

Theorem 4.8. Assume $m<0$. Let $f, \bar{f}$ and $g, \bar{g}$ such that (4.1), resp. (4.2), hold. Let $u$ and $\bar{u}$ be two solutions of problem (1.7) with data $(f, g)$, resp. $(\bar{f}, \bar{g})$. If $g \leq \bar{g}$, then

$$
\int_{\Omega}(u-\bar{u})^{+} d x \leq \int_{\Omega}(f-\bar{f})^{+} d x .
$$

In particular, the uniqueness part of Theorem 4.3 holds true.

Proof. Let $\mathbf{w}$ and $\overline{\mathbf{w}}$ be the gradient-director fields associated to $u$, resp. $\bar{u}$, and let $\mathbf{z}=u^{m} \mathbf{w}$, $\bar{z}=\bar{u}^{m} \overline{\mathbf{w}}$. We know that

$$
u-f=\operatorname{div} \mathbf{z} \quad \text { and } \quad \bar{u}-\bar{f}=\operatorname{div} \overline{\mathbf{z}} \quad \text { in } L^{\infty}(\Omega) .
$$

We also know, by Lemma 4.7, that (4.6) holds for both. Hence

$$
(\mathbf{w}-\overline{\mathbf{w}}, D u-D \bar{u}) \stackrel{(4.6)}{=}|D u|+|D \bar{u}|-(\mathbf{w}, D \bar{u})-(\overline{\mathbf{w}}, D u) \geq 0 \quad \text { as measures, }
$$

since $\|\mathbf{w}\|_{\infty} \leq 1$ and $\|\overline{\mathbf{w}}\|_{\infty} \leq 1$. Multiplying the equations in (4.39) by $T_{\varepsilon}(u-\bar{u})^{+}$, applying (2.5), and subtracting the two equalities we obtain

$$
\begin{aligned}
& \int_{\Omega}(u-\bar{u}+\bar{f}-f) T_{\varepsilon}(u-\bar{u})^{+} \mathrm{d} x \\
& \quad=-\int_{\Omega} \mathrm{d}\left(\mathbf{z}-\overline{\mathbf{z}}, D T_{\varepsilon}(u-\bar{u})^{+}\right)+\int_{\partial \Omega}[\mathbf{z}-\overline{\mathbf{z}}, \nu] T_{\varepsilon}(u-\bar{u})^{+} \mathrm{d} \mathcal{H}^{N-1} .
\end{aligned}
$$


Let us consider the first term on the right hand side of (4.41). Using the fact that the measure $D(u-\bar{u})$ is diffuse, we obtain

$$
\begin{aligned}
& -\int_{\Omega} \mathrm{d}\left(\mathbf{z}-\overline{\mathbf{z}}, D T_{\varepsilon}(u-\bar{u})^{+}\right) \\
& =\quad-\int_{\{0<u-\bar{u}<\varepsilon\}} \theta\left(\mathbf{z}-\overline{\mathbf{z}}, D T_{\varepsilon}(u-\bar{u})^{+}\right) \mathrm{d}\left|D T_{\varepsilon}(u-\bar{u})^{+}\right| \\
& \stackrel{(2.8)}{=}-\int_{\{0<u-\bar{u}<\varepsilon\}} \theta(\mathbf{z}-\overline{\mathbf{z}}, D(u-\bar{u})) \mathrm{d}|D(u-\bar{u})| \\
& =\quad-\int_{\{0<u-\bar{u}<\varepsilon\}} \mathrm{d}(\mathbf{z}-\overline{\mathbf{z}}, D(u-\bar{u})) \\
& \stackrel{(2.12)}{=}-\int_{\{0<u-\bar{u}<\varepsilon\}}\left(u^{m}-\bar{u}^{m}\right) \mathrm{d}(\mathbf{w}, D(u-\bar{u})) \\
& \quad-\int_{\{0<u-\bar{u}<\varepsilon\}} \bar{u}^{m} \mathrm{~d}(\mathbf{w}-\overline{\mathbf{w}}, D(u-\bar{u})) \\
& \stackrel{(4.40)}{\leq}-\int_{\{0<u-\bar{u}<\varepsilon\}}\left(u^{m}-\bar{u}^{m}\right) \mathrm{d}(\mathbf{w}, D(u-\bar{u})) .
\end{aligned}
$$

Since $u, \bar{u}$ are bounded above and below and the mapping $s \mapsto s^{m}$ is locally Lipschitz, a positive constant $C$, independent of $\varepsilon$, exists such that $\left|u^{m}-\bar{u}^{m}\right| \leq C|u-\bar{u}|$. Using also $\|\mathbf{w}\|_{\infty} \leq 1$ and the fact the measure $D(u-\bar{u})$ is diffuse, we see that

$$
\begin{aligned}
\left|\int_{\{0<u-\bar{u}<\varepsilon\}}\left(u^{m}-\bar{u}^{m}\right) \mathrm{d}(\mathbf{w}, D(u-\bar{u}))\right| & \leq C \varepsilon \int_{\{0<u-\bar{u}<\varepsilon\}} \mathrm{d}|D(u-\bar{u})| \\
& =C \varepsilon \int_{\Omega} \mathrm{d}\left|D T_{\varepsilon}(u-\bar{u})^{+}\right| .
\end{aligned}
$$

By the coarea formula [3, Theorem 3.40], we get

$$
\begin{aligned}
\int_{\Omega} \mathrm{d}\left|D T_{\varepsilon}(u-\bar{u})^{+}\right| & =\int_{-\infty}^{+\infty} P\left(\left\{T_{\varepsilon}(u-\bar{u})^{+}>\lambda\right\}\right) \mathrm{d} \lambda \\
& =\int_{0}^{\varepsilon} P(\{u-\bar{u}>\lambda\}) \mathrm{d} \lambda=o_{\varepsilon}(1) \quad \text { as } \varepsilon \rightarrow 0,
\end{aligned}
$$

since $\lambda \mapsto P(\{u-\bar{u}>\lambda\})$ is integrable on $\mathbb{R}$. Inserting (4.42), (4.43), and (4.44) into (4.41), dividing by $\varepsilon$, and letting $\varepsilon \rightarrow 0$, we obtain

$$
\int_{\Omega}(u-\bar{u}+\bar{f}-f) \operatorname{sign}_{0}^{+}(u-\bar{u}) \mathrm{d} x \leq \int_{\partial \Omega}([\mathbf{z}, \nu]-[\overline{\mathbf{z}}, \nu]) \operatorname{sign}_{0}^{+}(u-\bar{u}) \mathrm{d} \mathcal{H}^{N-1},
$$

where

$$
\operatorname{sign}_{0}^{+}(r)= \begin{cases}1 & \text { if } r>0 \\ 0 & \text { if } r \leq 0\end{cases}
$$

Since $u \leq g \leq \bar{g}$ in $\partial \Omega$,

$$
\{x \in \partial \Omega: u(x)>\bar{u}(x)\} \subseteq\{x \in \partial \Omega: \bar{g}(x)>\bar{u}(x)\} .
$$


By (2.7) and (4.5b), $[\mathbf{z}, \nu] \leq u^{m}$ and $[\overline{\mathbf{z}}, \nu]=\bar{u}^{m} \mathcal{H}^{N-1}$-a.e. on $\partial \Omega \cap\{u>\bar{u}\}$. Therefore

$$
\begin{aligned}
& \int_{\partial \Omega}([\mathbf{z}, \nu]-[\overline{\mathbf{z}}, \nu]) \operatorname{sign}_{0}^{+}(u-\bar{u}) \mathrm{d} \mathcal{H}^{N-1} \\
\leq & \int_{\partial \Omega}\left(u^{m}-\bar{u}^{m}\right) \operatorname{sign}_{0}^{+}(u-\bar{u}) \mathrm{d} \mathcal{H}^{N-1} \leq 0,
\end{aligned}
$$

and we conclude that

$$
\int_{\Omega}(u-\bar{u})^{+} \mathrm{d} x \leq \int_{\Omega}\left((f-\bar{f})^{+}-(f-\bar{f})^{-}\right) \operatorname{sign}_{0}^{+}(u-\bar{u}) \mathrm{d} x \leq \int_{\Omega}(f-\bar{f})^{+} \mathrm{d} x .
$$

\section{The degenerate case}

In this section we analyze the degenerate case of Problem (1.7), $m>0$. As we already mentioned, in contrast with the singular case, for $m>0$ it is natural to allow the data (hence, the solution) to become zero. This reflects into some technical complications in the proofs of both existence and uniqueness, since a priori bounds only guarantee that $T_{a}^{\infty}(u) \in B V(\Omega)$ for any $a>0$. Therefore, we will need some further properties of the space $T B V^{+}$, which are proved in the next subsection.

\subsection{Properties of the space $T B V^{+}(\Omega)$}

First of all, we argue that the trace of functions in $T B V^{+}(\Omega)$ is well defined.

Lemma 5.1. Let $\Omega$ be a bounded open set with Lipschitz boundary and $u \in T B V^{+}(\Omega)$. Then there exists $u^{\Omega} \in L^{1}(\partial \Omega ;[0,+\infty))$ such that

$$
\lim _{\rho \rightarrow 0} f_{\Omega \cap B_{\rho}\left(x_{0}\right)}\left|u(x)-u^{\Omega}\left(x_{0}\right)\right| \mathrm{d} x=0 \quad \text { for } \mathcal{H}^{N-1} \text {-a.e. } x_{0} \in \partial \Omega \text {. }
$$

Moreover,

$$
u^{\Omega}=\lim _{a \rightarrow 0^{+}}\left(T_{a}^{\infty}(u)\right)^{\Omega} \quad \mathcal{H}^{N-1} \text {-a.e. in } \partial \Omega
$$

and

$$
F\left(u^{\Omega}\right)=(F(u))^{\Omega} \quad \text { for all } F \in W_{a}^{1, \infty}
$$

(see (2.3) for the definition of $W_{a}^{1, \infty}$ ).

Proof. Since $u \in T B V^{+}(\Omega)$, we have $\left(T_{a}^{\infty}(u)\right)^{\Omega} \in L^{1}(\partial \Omega)$ for all $a>0$. Of course $0 \leq$ $\left(T_{a^{\prime}}^{\infty}(u)\right)^{\Omega} \leq\left(T_{a^{\prime \prime}}^{\infty}(u)\right)^{\Omega}$ for $0<a^{\prime}<a^{\prime \prime}$. Hence, by monotone convergence, the point-wise limit $u^{\Omega}(x)$ in (5.2) exists a.e. in $\partial \Omega$ and $u^{\Omega} \in L^{1}(\partial \Omega ;[0,+\infty))$. For a.e. $x_{0} \in \partial \Omega$, we have

$$
\begin{gathered}
f_{\Omega \cap B_{\rho}\left(x_{0}\right)}\left|u(x)-u^{\Omega}\left(x_{0}\right)\right| \mathrm{d} x \leq \lim _{\rho \rightarrow 0} f_{\Omega \cap B_{\rho}\left(x_{0}\right)}\left|u(x)-T_{a}^{\infty}(u(x))\right| \mathrm{d} x \\
+\lim _{\rho \rightarrow 0} f_{\Omega \cap B_{\rho}\left(x_{0}\right)}\left|T_{a}^{\infty}(u(x))-\left(T_{a}^{\infty}(u)\right)^{\Omega}\left(x_{0}\right)\right| \mathrm{d} x
\end{gathered}
$$




$$
+\lim _{\rho \rightarrow 0} f_{\Omega \cap B_{\rho}\left(x_{0}\right)}\left|\left(T_{a}^{\infty}(u)\right)^{\Omega}\left(x_{0}\right)-u^{\Omega}\left(x_{0}\right)\right| \mathrm{d} x .
$$

Noting that $\left|u(x)-T_{a}^{\infty}(u(x))\right|=(a-u) \chi_{\{u<a\}}<a$ and recalling (5.2), for any $\varepsilon>0$ we may find $a>0$ such that

$$
\begin{aligned}
& \limsup _{\rho \rightarrow 0} f_{\Omega \cap B_{\rho}\left(x_{0}\right)}\left|u(x)-u^{\Omega}\left(x_{0}\right)\right| \mathrm{d} x \\
& \leq \varepsilon+\limsup _{\rho \rightarrow 0} f_{\Omega \cap B_{\rho}\left(x_{0}\right)}\left|T_{a}^{\infty}(u(x))-\left(T_{a}^{\infty}(u)\right)^{\Omega}\left(x_{0}\right)\right| \mathrm{d} x,
\end{aligned}
$$

hence (5.1) follows from the arbitrariness of $\varepsilon$ and the definition of trace of $T_{a}^{\infty}(u)$. In order to prove (5.3), for $x_{0} \in \partial \Omega$ we write

$$
\begin{aligned}
\left|(F(u))^{\Omega}\left(x_{0}\right)-F\left(u^{\Omega}\left(x_{0}\right)\right)\right|= & \lim _{\rho \rightarrow 0} f_{\Omega \cap B_{\rho}\left(x_{0}\right)}\left|(F(u))^{\Omega}\left(x_{0}\right)-F\left(u^{\Omega}\left(x_{0}\right)\right)\right| \mathrm{d} x \\
\leq & \lim _{\rho \rightarrow 0} f_{\Omega \cap B_{\rho}\left(x_{0}\right)}\left|(F(u))^{\Omega}\left(x_{0}\right)-F(u(x))\right| \mathrm{d} x \\
& +\lim _{\rho \rightarrow 0} f_{\Omega \cap B_{\rho}\left(x_{0}\right)}\left|F(u(x))-F\left(u^{\Omega}\left(x_{0}\right)\right)\right| \mathrm{d} x \\
\leq & L \lim _{\rho \rightarrow 0} f_{\Omega \cap B_{\rho}\left(x_{0}\right)}\left|u(x)-u^{\Omega}\left(x_{0}\right)\right| \mathrm{d} x
\end{aligned}
$$

and the limit is zero because of (5.1).

In view of (5.3), hereafter we will omit the superscript $\Omega$. The next result is a version of Lemma 2.5 for $T B V^{+}$-functions:

Lemma 5.2. Let $u \in T B V^{+}(\Omega) \cap L^{\infty}(\Omega)$, w $\in L^{\infty}\left(\Omega ; \mathbb{R}^{N}\right)$ and $\mathbf{z}=u^{m} \mathbf{w} \in X(\Omega)$. Then

(i) For almost every $0<a<b \leq+\infty$, w $\chi_{\{a<u<b\}} \in X_{\mathcal{M}}(\Omega)$ and

$$
\begin{aligned}
& {[\mathbf{z}, \nu] \chi_{\{a<u<b\}}=T_{a}^{b}(u)^{m}\left[\mathbf{w} \chi_{\{a<u<b\}}, \nu\right] \mathcal{H}^{N-1} \text {-a.e. on } \partial \Omega,} \\
& {\left[\mathbf{z}, \nu_{T_{a}^{b}(u)}\right]^{ \pm} \chi_{\{a<u<b\}}=\left(T_{a}^{b}(u)^{m}\right)^{ \pm}\left[\mathbf{w}_{\{a<u<b\}}, \nu_{T_{a}^{b}(u)}\right]^{ \pm},} \\
& \mathcal{H}^{N-1} \text {-a.e. on } J_{T_{a}^{b}(u)} \text {; }
\end{aligned}
$$

(ii)

$$
\mathcal{H}^{N-1}\{x \in \partial \Omega: u(x)=0,[\mathbf{z}, \nu](x) \neq 0\}=0,
$$

Proof. Since $u \in T B V^{+}(\Omega) \cap L^{\infty}(\Omega), u^{-m} \chi_{\{a<u<b\}} \in B V(\Omega) \cap L^{\infty}(\Omega)$ for almost any $0<$ $a<b \leq+\infty$. Therefore, it follows from Lemma 2.2 (applied with $u^{-m} \chi_{\{a<u<b\}}$ in place of $u$ ) that $\mathbf{w} \chi_{\{a<u<b\}} \in X_{\mathcal{M}}(\Omega)$ and (5.4) holds. By the same argument, (5.5) follows immediately from (2.11).

Let us prove (ii). Let $\varphi$ be a non-negative mollifier and $\varphi_{\rho}(x)=\rho^{-N} \varphi\left(\left(x-x_{0}\right) / \rho\right)$. Then, for $\mathcal{H}^{N-1}$-a.e. $x_{0} \in \partial \Omega$ we have

$$
\begin{aligned}
{[\mathbf{z}, \nu]\left(x_{0}\right) } & =C \lim _{\rho \rightarrow 0} \rho \int_{\partial \Omega} \varphi_{\rho}[\mathbf{z}, \nu] \mathrm{d} \mathcal{H}^{N-1} \\
& =C \lim _{\rho \rightarrow 0}\left(\rho \int_{\Omega} \mathbf{z} \cdot \nabla \varphi_{\rho} \mathrm{d} x+\rho \int_{\Omega} \varphi_{\rho} \operatorname{div} \mathbf{z} \mathrm{d} x\right) .
\end{aligned}
$$


The second integral on the r.h.s. vanishes in the limit since $\operatorname{div} \mathbf{z} \in L^{\infty}(\Omega)$. For the first one, since $|\mathbf{z}| \leq u^{m}$ and $\left|\nabla \varphi_{\rho}\right| \leq C \rho^{-N-1} \chi_{B_{\rho}\left(x_{0}\right)}$, for a.e. $x_{0} \in \partial \Omega$ we have

$$
\begin{aligned}
\limsup _{\rho \rightarrow 0} \rho \int_{\Omega} \mathbf{z} \cdot \nabla \varphi_{\rho} \mathrm{d} x & \leq C \limsup _{\rho \rightarrow 0} f_{\Omega \cap B_{\rho}\left(x_{0}\right)} u^{m} \mathrm{~d} x \\
& \leq C\|u\|_{L^{\infty}(\Omega)}^{m-1} \lim _{\rho \rightarrow 0} f_{\Omega \cap B_{\rho}\left(x_{0}\right)} u \mathrm{~d} x=C\|u\|_{L^{\infty}(\Omega)}^{m-1} u\left(x_{0}\right),
\end{aligned}
$$

hence $[\mathbf{z}, \nu]\left(x_{0}\right)=0 \mathcal{H}^{N-1}$-a.e. on $\{u=0\} \cap \partial \Omega$.

The last auxiliary result we need shows that, as intuition suggests, in case $u \in D T B V^{+}(\Omega)$, pairings of the form $(\mathbf{z}, D T(u))$ are oblivious to the values of $\mathbf{z}$ outside $\operatorname{supp}\left(T^{\prime}\right)$.

Lemma 5.3. Let $u \in D T B V^{+}(\Omega) \cap L^{\infty}(\Omega)$ and $\mathbf{z} \in X_{\mathcal{M}}(\Omega)$. Then $\mathbf{z} \chi_{\{a<u<b\}} \in X_{\mathcal{M}}(\Omega)$ for a.e. $0<a<b \leq+\infty$ and

$$
\left(\mathbf{z}, D T_{a}^{b}(u)\right)=\left(\mathbf{z} \chi_{\{a<u<b\}}, D T_{a}^{b}(u)\right) \text { for a.e. } a<b \leq+\infty .
$$

Proof. Since $\chi_{\{a<u<b\}} \in B V(\Omega) \cap L^{\infty}(\Omega)$ for a.e. $a>0$ and a.e. $a<b \leq+\infty$, it follows from Lemma 2.2 that $\mathbf{z}_{\{a<u<b\}} \in X_{\mathcal{M}}(\Omega)$ for a.e. $a>0$ and $a<b \leq+\infty$. We first prove (5.7) for $b=+\infty$, i.e.,

$$
\left(\mathbf{z}, D T_{a}^{\infty}(u)\right)=\left(\mathbf{z} \chi_{\{u>a\}}, D T_{a}^{\infty}(u)\right) \text { for a.e. } a>0 .
$$

We let $\bar{T}(s):=T_{a}^{\infty}(s)-a$ and we note that

$$
D T_{a}^{\infty}(u)=D \bar{T}(u), \quad \bar{T}(u)=\bar{T}(u) \chi_{\{u>a\}} .
$$

Then

$$
\begin{array}{rll}
\left(\mathbf{z} \chi_{\{u>a\}}, D T_{a}^{\infty}(u)\right) & \stackrel{(5.9)}{=} & \left(\mathbf{z} \chi_{\{u>a\}}, D \bar{T}(u)\right) \\
& \stackrel{(2.6)}{=} & \operatorname{div}\left(\mathbf{z} \chi_{\{u>a\}} \bar{T}(u)\right)-\bar{T}(u) \operatorname{div}\left(\mathbf{z} \chi_{\{u>a\}}\right) \\
& \stackrel{(2.6),(5.9)}{=} & \operatorname{div}(\mathbf{z} \bar{T}(u))-\bar{T}(u) \operatorname{div} \mathbf{z}-\bar{T}(u)\left(\mathbf{z}, D \chi_{\{u>a\}}\right) \\
& \stackrel{(2.6)}{=} & (\mathbf{z}, D \bar{T}(u))-\bar{T}(u)\left(\mathbf{z}, D \chi_{\{u>a\}}\right) .
\end{array}
$$

Note that $\left(\mathbf{z}, D \chi_{\{u>a\}}\right) \ll\left|D \chi_{\{u>a\}}\right|$ and $\bar{T}(u)=0\left|D \chi_{\{u>a\}}\right|$-a.e. (since $\mathcal{H}^{N-1}\left(S_{u}^{*}\right)=0$ ). Therefore, $\bar{T}(u)\left(\mathbf{z}, D \chi_{\{u>a\}}\right)=0$ and the conclusion follows using again (5.9).

We now prove the statement for a generic $b<+\infty$. The argument is the same, but exploits (5.8). We note that

$$
T_{a}^{b}(s)=T_{a}^{\infty}\left(T_{b}(s)\right)=T_{b}\left(T_{a}^{\infty}(s)\right) \quad \text { for all } s \geq 0
$$

Therefore

$$
\left(\mathbf{z} \chi_{\{a<u<b\}}, D T_{a}^{b}(u)\right) \stackrel{(5.10)}{=}\left(\mathbf{z} \chi_{\{u>a\}} \chi_{\{u<b\}}, D T_{b}\left(T_{a}^{\infty}(u)\right)\right)=\left(\overline{\mathbf{z}} \chi_{\{u<b\}}, D \bar{T}(u)\right),
$$

where $\overline{\mathbf{z}}=\mathbf{z} \chi_{\{u>a\}} \bar{T}(u)=T_{b}\left(T_{a}^{\infty}(u)\right)-b$. Noting that $\bar{T}(u)=\bar{T}(u) \chi_{\{u<b\}}$ and arguing exactly as above, we obtain

$$
\left(\mathbf{z} \chi_{\{a<u<b\}}, D T_{a}^{b}(u)\right)=(\overline{\mathbf{z}}, D \bar{T}(u))-\bar{T}(u)\left(\overline{\mathbf{z}}, D \chi_{\{u<b\}}\right)=(\overline{\mathbf{z}}, D \bar{T}(u)),
$$


where in the last equality we have used that $\left(\overline{\mathbf{z}}, D \chi_{\{u<b\}}\right) \ll\left|D \chi_{\{u<b\}}\right|$ and that $\bar{T}(u)=0$ $\left|D \chi_{\{u<b\}}\right|$-a.e. (here we use again that $\mathcal{H}^{N-1}\left(S_{u}^{*}\right)=0$ ). Therefore, recalling the definition of $\overline{\mathbf{z}}$ and $\bar{T}$,

$$
\left(\mathbf{z} \chi_{\{a<u<b\}}, D T_{a}^{b}(u)\right)=\left(\mathbf{z} \chi_{\{u>a\}}, D T_{a}^{\infty}\left(T_{b}(u)\right)\right) \stackrel{(5.10)}{=}\left(\mathbf{z}, D T_{a}^{b}(u)\right) .
$$

\subsection{Existence}

We can now look at the existence of a solution to (1.7) in the case $m>0$. We assume:

$$
0 \leq f \in L^{\infty}(\Omega), \quad 0 \leq g \in L^{\infty}(\partial \Omega)
$$

We introduce the following notion of solution.

Definition 5.4. Assume $m>0$ and (5.11). A function $u: \Omega \rightarrow[0,+\infty)$ is a solution of problem (1.7) with data $(f, g)$ if $u \in T B V^{+}(\Omega) \cap L^{\infty}(\Omega)$ and there exist $\mathbf{w} \in L^{\infty}\left(\Omega ; \mathbb{R}^{N}\right)$ such that $\|\mathbf{w}\|_{\infty} \leq 1$ and $\mathbf{z}:=u^{m} \mathbf{w} \in X(\Omega)$ satisfies

$$
\begin{gathered}
|D \phi(T(u))| \leq(\mathbf{z}, D T(u)) \quad \text { as measures for any } T \in \mathcal{T}, \\
u-f=\operatorname{div} \mathbf{z} \quad \text { in } \mathcal{D}^{\prime}(\Omega)
\end{gathered}
$$

and

$$
\begin{gathered}
u \geq g \mathcal{H}^{N-1} \text {-a.e. on } \partial \Omega, \\
{[\mathbf{z}, \nu]=-u^{m} \text { if } u>g \quad \mathcal{H}^{N-1} \text {-a.e. on } \partial \Omega .}
\end{gathered}
$$

Remark 5.5. The boundary conditions are consistent with those used in [8] for equation (1.4) with $m=1$.

Definition 5.4 differs from Definition 4.1 since we allow data (and therefore solutions) to become zero: since the equation degenerates, we have little control at $u=0$ and we need to use truncation functions. For data which are bounded away from zero this new formulation is not needed and well-posedness can be obtained as in the previous section with minor modifications. Indeed, if there exists $C>0$ such that $C \leq f(x)$ for a.e. $x \in \Omega$ and $C \leq g(x)$, for a. e. $x \in \partial \Omega$, it is straightforward to see that $v \equiv C$ is a subsolution to (3.2). Therefore the approximate solutions, whence the limiting solutions obtained in Lemma 5.7 below, are strictly positive.

The main result of this section is the following.

Theorem 5.6. Assume $m>0$ and (5.11). Then there exists a unique solution $u$ of (1.7) with data $(f, g)$ in the sense of Definition 5.4. Furthermore, $u \in D T B V^{+}(\Omega)$,

$$
\left(\mathbf{w}, D T_{a}^{b}(u)\right)=\left|D T_{a}^{b}(u)\right| \text { for a.e. } 0<a<b \leq+\infty,
$$

and

$$
\left(\mathbf{z}, D T_{a}^{b}(u)\right)=\left|D \phi\left(T_{a}^{b}(u)\right)\right| \quad \text { for a.e. } 0<a<b \leq+\infty .
$$

In proving existence of a solution, we will follow the arguments used in the singular case highlighting only the main differences, which are related to the need of using truncation functions. 
Lemma 5.7. Assume $m>0$ and (5.11). Then there exists a pair $(u, \mathbf{w})$ such that $u \in T B V^{+}(\Omega) \cap$ $L^{\infty}(\Omega)$ and $\mathbf{w} \in L^{\infty}\left(\Omega ; \mathbb{R}^{N}\right)$ with $\|\mathbf{w}\|_{\infty} \leq 1$, such that $\mathbf{z}=u^{m} \mathbf{w} \in X(\Omega)$, and

$$
u-f=\operatorname{div} \mathbf{z} \quad \text { in } \mathcal{D}^{\prime}(\Omega) .
$$

Furthermore,

$$
\left|D \phi_{F}(T(u))\right| \leq(\mathbf{z}, D F(T(u))) \quad \text { as measures, }
$$

and

$$
\left|\phi_{F}(T(g))-\phi_{F}(T(u))\right| \leq(F(T(g))-F(T(u)))[\mathbf{z}, \nu] \quad \mathcal{H}^{N-1} \text {-a.e. on } \partial \Omega
$$

for any $T \in \mathcal{T}$ and any nondecreasing $F \in W_{l o c}^{1, \infty}((0,+\infty))$, with $\phi_{F}$ as in (2.1).

Proof. Arguments are analogous to those of Lemma 4.5. Let $\tilde{g}$ be a function in $H^{1}\left(\Omega ;\left[0,\|g\|_{L^{\infty}(\partial \Omega)}\right]\right)$ such that $\tilde{g}=g$ on $\partial \Omega$. Again, for simplicity, we agree that $\int f \mathrm{~d} \mu=\int_{\Omega} f \mathrm{~d} \mu, \int f=\int f \mathrm{~d} x$, and $C \geq 1$ denotes a generic constant independent of $\varepsilon \in(0,1)$. Let $u_{\varepsilon} \in H^{1}(\Omega) \cap L^{\infty}(\Omega)$ be a solution of (3.2) as given by Lemma 3.1. We recall that

$$
0 \leq u_{\varepsilon} \leq \max \left\{\|f\|_{L^{\infty}(\Omega)},\|g\|_{L^{\infty}(\partial \Omega)}\right\} .
$$

Testing the equation (3.2) by $u_{\varepsilon}-\tilde{g}$ and using that $\varepsilon^{m} \leq\left(\varepsilon+u_{\varepsilon}\right)^{m} \leq C$, we get

$$
\int u_{\varepsilon}^{2}+\int u_{\varepsilon}^{m}\left|\nabla u_{\varepsilon}\right|+\frac{\varepsilon}{2} \int\left|\nabla u_{\varepsilon}\right|^{2} \leq C\left(1+\int f^{2}+\int \tilde{g}^{2}+\int|\nabla \tilde{g}|^{2}\right)
$$

and since

$$
\int_{\Omega}\left|\nabla T_{a}^{\infty}\left(u_{\varepsilon}\right)\right| \leq \int_{\left\{a \leq u_{\varepsilon}\right\}}\left|\nabla u_{\varepsilon}\right| \leq \frac{1}{a^{m}} \int_{\left\{a \leq u_{\varepsilon}\right\}} u_{\varepsilon}^{m}\left|\nabla u_{\varepsilon}\right| \leq \frac{1}{a^{m}} \int_{\Omega} u_{\varepsilon}^{m}\left|\nabla u_{\varepsilon}\right|,
$$

we conclude that

$$
\int u_{\varepsilon}^{2}+a^{m} \int\left|\nabla T_{a}^{\infty}\left(u_{\varepsilon}\right)\right|+\int\left|\nabla\left(u_{\varepsilon}^{m+1}\right)\right|+\varepsilon \int\left|\nabla u_{\varepsilon}\right|^{2} \leq C .
$$

Because of (5.20) and (5.19), there exist $u \in T B V^{+}(\Omega) \cap L^{\infty}(\Omega)$ and $\mathbf{w} \in L^{\infty}\left(\Omega ; \mathbb{R}^{N}\right)$ such that (up to subsequences, not relabeled)

$$
\begin{aligned}
u_{\varepsilon} \stackrel{*}{\rightarrow} u & \text { in } T B V^{+}(\Omega) \text { and in } L^{\infty}(\Omega) \\
u_{\varepsilon} \rightarrow u & \mathcal{L}^{N} \text {-a.e. and in } L^{p}(\Omega) \text { for all } p<+\infty \\
u_{\varepsilon}^{m+1} \stackrel{*}{\rightarrow} u^{m+1} & \text { in } B V(\Omega) \\
\varepsilon \nabla u_{\varepsilon} \rightarrow 0 & \text { strongly in } L^{2}\left(\Omega ; \mathbb{R}^{N}\right) \\
\nabla u_{\varepsilon} /\left|\nabla u_{\varepsilon}\right|_{\varepsilon}=: \mathbf{w}_{\varepsilon} \stackrel{*}{\rightarrow} \mathbf{w} & \text { in } L^{\infty}\left(\Omega ; \mathbb{R}^{N}\right) \\
\left(\varepsilon+u_{\varepsilon}\right)^{m} \mathbf{w}_{\varepsilon}=: \tilde{\mathbf{z}}_{\varepsilon} \stackrel{*}{\rightarrow} \mathbf{z}=u^{m} \mathbf{w} & \text { in } L^{\infty}\left(\Omega ; \mathbb{R}^{N}\right),
\end{aligned}
$$

and (5.21) and (5.22) combine into

$$
\tilde{\mathbf{z}}_{\varepsilon}+\varepsilon \nabla u_{\varepsilon}=: \mathbf{z}_{\varepsilon} \rightarrow \mathbf{z} \quad \text { in } L^{2}\left(\Omega ; \mathbb{R}^{N}\right) .
$$

Passing to the limit as $\varepsilon \rightarrow 0$ in the approximating equations we obtain (5.16).

The proof of (5.17) and (5.18) is a straightforward adaptation of that of (4.14) and (4.15), testing (3.2) by $\varphi\left(F\left(T\left(u_{\varepsilon}\right)\right)-F(T(\tilde{g}))\right)$ with $0 \leq \varphi \in C^{\infty}(\Omega)$. Therefore we omit the details. 
We have the following:

Lemma 5.8. Assume $m>0$ and (5.11). Let $u, \mathrm{w}$ be as in Lemma 5.7. Then

$$
u \geq g \quad \mathcal{H}^{N-1} \text {-a.e. on } \partial \Omega .
$$

Furthermore,

$$
[\mathbf{z}, \nu]=-u^{m} \quad \text { if } u>g \quad \mathcal{H}^{N-1} \text {-a.e. on } \partial \Omega .
$$

Proof. The proof is analogous to the one of Lemma 4.6, hence we only show the main differences. For notational convenience, we let $T=T_{a}^{b} \in \mathcal{T}$. For (5.23), applying (5.18) with $F(s)=s$, we see that

$$
\frac{1}{m+1}\left|(T(g))^{m+1}-(T(u))^{m+1}\right| \leq(T(g)-T(u))[\mathbf{z}, \nu] \quad \mathcal{H}^{N-1} \text {-a.e. on } \partial \Omega .
$$

We now argue for a fixed $x \in \partial \Omega$ and up to $\mathcal{H}^{N-1}$-negligible sets. If $u(x)=0$ at some point $x \in \partial \Omega$, it follows from (5.6) that $[\mathbf{z}, \nu]=0$. Hence (5.25) implies that $(T(g))^{m+1}=a^{m+1}$ for all $T \in \mathcal{T}$ : therefore $g(x)=0$ and (5.23) holds. If instead $u(x) \neq 0$, let $a$ and $b$ such that $a<u(x)<b$. We have

$$
\begin{aligned}
& \frac{1}{m+1}\left|T(g)^{m+1}-T(u)^{m+1}\right| \leq(T(g)-T(u))[\mathbf{z}, \nu] \\
& \stackrel{(5.4)}{\leq} T(u)^{m}|T(g)-T(u)|,
\end{aligned}
$$

which implies (5.23) arguing as in the proof of (4.30).

In order to prove (5.24), let $F(s)=\frac{s^{m p}}{p}$ and let us fix $x \in \partial \Omega$ such that (5.18) holds true. Then $\phi_{F}(T(s))=\frac{T(s)^{m(p+1)}-1}{p+1}$ and we have

$$
\left|\frac{(T(u))^{m(p+1)}-(T(g))^{m(p+1)}}{p+1}\right| \leq \frac{\left((T(g))^{m p}-(T(u))^{m p}\right)}{p}[\mathbf{z}, \nu] .
$$

The rest of the proof is similar to that of (4.31) and we omit it.

The existence part of Theorem 5.6 is an immediate consequence of the previous lemmas:

Proof of Theorem 5.6, existence. Lemma 5.7 gives the existence of a function $u \in T B V^{+}(\Omega) \cap$ $L^{\infty}(\Omega)$, and $\mathbf{w} \in L^{\infty}\left(\Omega ; \mathbb{R}^{N}\right)$ with $\|\mathbf{w}\|_{\infty} \leq 1$ such that $\mathbf{z}=u^{m} \mathbf{w} \in X(\Omega)$ and (5.12) and (5.13) are satisfied. The boundary datum $g$ is achieved in the sense of Definition 5.4 thanks to Lemma 5.8 .

\subsection{Regularity}

In the next two Lemmas, we show that any solution to (1.7) in the sense of Definition 5.4 has the additional regularity properties stated in Theorem 5.6. First we show that, as in the singular case, solutions' gradients have no jump part. 
Lemma 5.9. Assume $m>0$ and (5.11). Let $u \in T B V^{+}(\Omega) \cap L^{\infty}(\Omega)$ and $\mathbf{w} \in L^{\infty}(\Omega)$ be such that $\mathbf{z}=u^{m} \mathbf{w} \in X(\Omega),\|\mathbf{w}\|_{\infty} \leq 1$ and

$$
\left|D^{j} \phi(T(u))\right| \leq(\mathbf{z}, D T(u))\left\llcorner J_{T(u)} \quad\right. \text { as measures }
$$

for any $T \in \mathcal{T}^{\infty}$. Then $u \in D T B V^{+}(\Omega)$.

Proof. Let $T=T_{a}^{\infty}, a>0$, and recall that $\phi(s)=\frac{s^{m+1}}{m+1}$. Note that $J_{T(u)}=J_{\phi(T(u))}$ and $\nu_{T(u)}=\nu_{T(u)^{m}}$ on $J_{T(u)}$. Since $\mathbf{z} \in X(\Omega)$,

$$
(\operatorname{div} \mathbf{z})\left\llcorner J_{T(u)}=0\right.
$$

and

$$
\left[\mathbf{z}, \nu_{T(u)}\right]^{+\stackrel{(2.10)}{=}}\left[\mathbf{z}, \nu_{T(u)}\right]^{-}=: \Psi \quad \mathcal{H}^{N-1} \text {-a.e. on } J_{T(u)} .
$$

Therefore, by (5.5), for almost every $a>0$,

$$
|\Psi| \chi_{\{u>a\}} \leq \min \left\{\left(T(u)^{m}\right)^{+},\left(T(u)^{m}\right)^{-}\right\} \quad \mathcal{H}^{N-1} \text {-a.e. on } J_{T(u)} .
$$

We have

$$
\begin{aligned}
& \left|D^{j} \phi(T(u))\right| \stackrel{(5.28)}{\leq} \quad(\mathbf{z}, D T(u))\left\llcorner J_{T(u)}\right. \\
& \stackrel{(2.6)}{=}\left(-T(u)^{*} \operatorname{div} \mathbf{z}+\operatorname{div}(T(u) \mathbf{z})\right)\left\llcorner J_{T(u)}\right. \\
& \stackrel{(5.29)}{=} \operatorname{div}(T(u) \mathbf{z})\left\llcorner J_{T(u)}\right.
\end{aligned}
$$

Arguing as in the proof of Lemma 4.7, Lemmas 2.4-2.5 and (5.32) imply that $T(u)^{+}=T(u)^{-}$. Therefore, $0=\mathcal{H}^{N-1}\left(J_{T(u)}\right)=\mathcal{H}^{N-1}\left(S_{T(u)}\right)$ for almost every $a>0$ : by Lemma $2.1, \mathcal{H}^{N-1}\left(S_{u}^{*}\right)=$ 0 and the proof is complete.

Lemma 5.10. Let $u \in D T B V^{+}(\Omega) \cap L^{\infty}(\Omega)$ and $\mathbf{w} \in X_{\mathcal{M}}(\Omega)$ be such that $\mathbf{z}=u^{m} \mathbf{w} \in X(\Omega)$, $\|\mathbf{w}\|_{\infty} \leq 1$ and (5.12) holds. Then (5.15) holds.

Proof. Letting $T=T_{a}^{b}$, we notice that

$$
u=T(u)|D T(u)| \text {-a.e. } \quad \text { and } \quad\left(\mathbf{w} \chi_{\{a<u<b\}}, D(T(u))\right) \ll|D T(u)| .
$$

Therefore

$$
\begin{aligned}
|D \phi(T(u))| \stackrel{(5.12)}{\leq} & (\mathbf{z}, D T(u)) \stackrel{(5.7)}{=}\left(\mathbf{z} \chi_{\{a<u<b\}}, D T(u)\right) \\
\stackrel{(2.12)}{=} & u^{m}\left(\mathbf{w} \chi_{\{a<u<b\}}, D T(u)\right) \\
\stackrel{(5.33)}{=} & T(u)^{m}\left(\mathbf{w} \chi_{\{a<u<b\}}, D T(u)\right) \\
& =T(u)^{m} \theta\left(\mathbf{w} \chi_{\{a<u<b\}}, D T(u)\right)|D T(u)| \\
& =\theta\left(\mathbf{w} \chi_{\{a<u<b\}}, D T(u)\right)|D \phi(T(u))| \\
& \leq|D \phi(T(u))|,
\end{aligned}
$$

where in the last equality we have used the fact that $\mathcal{H}^{N-1}\left(S_{u}^{*}\right)=0$. Hence (5.15b) holds and $\theta\left(\mathbf{w} \chi_{\{a<u<b\}}, D(T(u))\right)=1|D T(u)|$-a.e., whence (5.15a). 


\subsection{Comparison and uniqueness}

The uniqueness part of Theorem 5.6 is an immediate consequence of the following comparison principle.

Theorem 5.11. Assume $m>0$ and $f, \bar{f}$ and $g, \bar{g}$ such that (5.11) holds. Let $u, \bar{u} \in D T B V^{+}(\Omega) \cap$ $L^{\infty}(\Omega)$ be two solutions of problem (1.7) with data $(f, g)$, resp. $(\bar{f}, \bar{g})$. If $g \leq \bar{g}$, then

$$
\int_{\Omega}(u-\bar{u})^{+} \leq \int_{\Omega}(f-\bar{f})^{+}
$$

In particular, the uniqueness part of Theorem 5.6 holds true.

Proof. Let $\mathbf{w}$, resp. $\overline{\mathbf{w}}$, and $\mathbf{z}$, resp. $\overline{\mathbf{z}}$, be as in Definition 5.4 for $u$, resp. $\bar{u}$. In particular,

$$
u-f=\operatorname{div} \mathbf{z} \quad \text { and } \quad \bar{u}-\bar{f}=\operatorname{div} \overline{\mathbf{z}} \quad \text { in } L^{\infty}(\Omega) .
$$

In addition, it follows from Lemmas 5.9 and 5.10 that $u, \bar{u} \in D T B V^{+}(\Omega)$ and that (5.15) holds for both pairs. Consequently, (5.15a) and Lemma 5.3 imply that

$$
\left.\begin{array}{l}
\left|D T_{a}^{b}(u)\right|=\left(\mathbf{w} \chi_{\{a<u<b\}}, D T_{a}^{b}(u)\right) \\
\left|D T_{a}^{b}(\bar{u})\right|=\left(\overline{\mathbf{w}} \chi_{\{a<\bar{u}<b\}}, D T_{a}^{b}(\bar{u})\right)
\end{array}\right\} \quad \text { for a.e. } 0<a<b \leq+\infty .
$$

Given $b>a>0$, we let

$$
T(r):=T_{a}^{b}(r)-a \quad \text { and } \quad T_{a, \varepsilon}(u, \bar{u}):=T_{\varepsilon}\left(\left(T_{a}^{\infty}(u)-T_{a}^{\infty}(\bar{u})\right)_{+}\right) .
$$

We multiply $(5.34)_{1}$ by $T(u) T_{a, \varepsilon}(u, \bar{u})$ and $(5.34)_{2}$ by $T(\bar{u}) T_{a, \varepsilon}(u, \bar{u})$, integrate by parts, and subtract both identities. Then,

$$
\begin{aligned}
\int_{\Omega}((u-f) T(u)-(\bar{u}-\bar{f}) T(\bar{u})) T_{a, \varepsilon}(u, \bar{u}) \mathrm{d} x \\
=\quad-\int_{\Omega} \mathrm{d}\left(\left(\mathbf{z}, D\left(T(u) T_{a, \varepsilon}(u, \bar{u})\right)-\left(\overline{\mathbf{z}}, D\left(T(\bar{u}) T_{a, \varepsilon}(u, \bar{u})\right)\right.\right.\right. \\
\quad+\int_{\partial \Omega} T_{a, \varepsilon}(u, \bar{u})(T(u)[\mathbf{z}, \nu]-T(\bar{u})[\overline{\mathbf{z}}, \nu]) \mathrm{d} \mathcal{H}^{N-1} \\
\stackrel{(2.13)}{=}-\int_{\Omega} T_{a, \varepsilon}(u, \bar{u}) \mathrm{d}((\mathbf{z}, D(T(u)+a))-(\overline{\mathbf{z}}, D T(\bar{u}))) \\
\quad-\int_{\Omega}\left(T(u) \mathrm{d}\left(\mathbf{z}, D T_{a, \varepsilon}(u, \bar{u})\right)-T(\bar{u}) \mathrm{d}\left(\overline{\mathbf{z}}, D T_{a, \varepsilon}(u, \bar{u})\right)\right) \\
\quad+\int_{\partial \Omega} T_{a, \varepsilon}(u, \bar{u})(T(u)[\mathbf{z}, \nu]-T(\bar{u})[\overline{\mathbf{z}}, \nu]) d \mathcal{H}^{N-1} \\
=: \quad I_{1}+I_{2}+I_{3} .
\end{aligned}
$$

As to $I_{1}$, we have

$$
I_{1} \stackrel{(5.15 \mathrm{~b})}{\leq} \int_{\Omega} T_{a, \varepsilon}(u, \bar{u}) \mathrm{d}(\overline{\mathbf{z}}, D T(\bar{u})) \stackrel{(5.36)}{\leq} \varepsilon \int_{\Omega} \mathrm{d}(\overline{\mathbf{z}}, D T(\bar{u}))
$$

As to $I_{2}$, by Lemma 2.6 and since $\mathbf{z} T(u)=\mathbf{w} u^{m} T(u) \chi_{\{u>a\}}$, we have

$$
\begin{aligned}
T(u)\left(\mathbf{z}, D T_{a, \varepsilon}(u, \bar{u})\right) & =\left(\mathbf{z} T(u), D T_{a, \varepsilon}(u, \bar{u})\right) \\
& =T(u) u^{m}\left(\mathbf{w} \chi_{\{u>a\}}, D T_{a, \varepsilon}(u, \bar{u})\right) .
\end{aligned}
$$


Similarly,

$$
T(\bar{u})\left(\overline{\mathbf{z}}, D T_{a, \varepsilon}(u, \bar{u})\right)=T(\bar{u}) \bar{u}^{m}\left(\overline{\mathbf{w}} \chi_{\{\bar{u}>a\}}, D T_{a, \varepsilon}(u, \bar{u})\right) .
$$

Then, since $\mathcal{H}^{N-1}\left(J_{T(u) u^{m}}^{*}\right)=0$ and $\left(\overline{\mathbf{w}} \chi_{\{\bar{u}>a\}}, D T_{a, \varepsilon}(u, \bar{u})\right) \ll\left|D T_{a, \varepsilon}(u, \bar{u})\right|$, we can add and subtract $T(u) u^{m} \mathrm{~d}\left(\overline{\mathbf{w}} \chi_{\{\bar{u}>a\}}, D T_{a, \varepsilon}(u, \bar{u})\right)$ to $I_{2}$ to get

$$
\begin{aligned}
I_{2}= & -\int_{\Omega} T(u) u^{m} \mathrm{~d}\left(\mathbf{w} \chi_{\{u>a\}}-\overline{\mathbf{w}} \chi_{\{\bar{u}>a\}}, D T_{a, \varepsilon}(u, \bar{u})\right) \\
& +\int_{\Omega}\left(T(u) u^{m}-T(\bar{u}) \bar{u}^{m}\right) \mathrm{d}\left(\overline{\mathbf{w}} \chi_{\{\bar{u}>a\}}, D T_{a, \varepsilon}(u, \bar{u})\right) \\
=: & I_{2,1}+I_{2,2} .
\end{aligned}
$$

As to $I_{2,1}$, using Lemma 5.2 we deduce that both $\mathbf{w} \chi_{\{u>a\}}$ and $\overline{\mathbf{w}} \chi_{\{\bar{u}>a\}}$ belong to $X_{\mathcal{M}}(\Omega)$, so that we have

$$
\begin{aligned}
& \left(\mathbf{w} \chi_{\{u>a\}}-\overline{\mathbf{w}} \chi_{\{\bar{u}>a\}}, D T_{a, \varepsilon}(u, \bar{u})\right) \\
& \stackrel{(2.9)}{=} \chi_{\left\{0<T_{a}^{\infty}(u)-T_{a}^{\infty}(\bar{u})<\varepsilon\right\}}\left(\mathbf{w} \chi_{\{u>a\}}-\overline{\mathbf{w}} \chi_{\{\bar{u}>a\}}, D\left(T_{a}^{\infty}(u)-T_{a}^{\infty}(\bar{u})\right)\right)
\end{aligned}
$$

and

$$
\begin{aligned}
& \left(\mathbf{w} \chi_{\{u>a\}}-\overline{\mathbf{w}} \chi_{\{\bar{u}>a\}}, D\left(T_{a}^{\infty}(u)-T_{a}^{\infty}(\bar{u})\right)\right) \\
& \stackrel{(5.35)}{=}\left|D T_{a}^{\infty}(u)\right|+\left|D T_{a}^{\infty}(\bar{u})\right|-\left(\mathbf{w} \chi_{\{u>a\}}, D T_{a}^{\infty}(u)\right)-\left(\overline{\mathbf{w}} \chi_{\{\bar{u}>a\}}, D T_{a}^{\infty}(\bar{u})\right) \\
& \geq 0 \quad(\text { since }\|\mathbf{w}\| \leq 1 \text { and }\|\overline{\mathbf{w}}\| \leq 1),
\end{aligned}
$$

hence

$$
I_{2,1} \leq 0
$$

As to $I_{2,2}$, again in view of Lemma 5.2, we have

$$
\begin{aligned}
& \left|\left(\overline{\mathbf{w}} \chi_{\{\bar{u}>a\}}, D T_{a, \varepsilon}(u, \bar{u})\right)\right| \\
& \quad \stackrel{(2.9)}{=} \chi_{\left\{0<T_{a}^{\infty}(u)-T_{a}^{\infty}(\bar{u})<\varepsilon\right\}}\left|\left(\overline{\mathbf{w}} \chi_{\{\bar{u}>a\}}, D\left(T_{a}^{\infty}(u)-T_{a}^{\infty}(\bar{u})\right)\right)\right| \\
& \quad \leq \quad \chi_{\left\{0<T_{a}^{\infty}(u)-T_{a}^{\infty}(\bar{u})<\varepsilon\right\}}\left|D\left(T_{a}^{\infty}(u)-T_{a}^{\infty}(\bar{u})\right)\right|
\end{aligned}
$$

and

$$
\begin{aligned}
& \chi_{\left\{0<T_{a}^{\infty}(u)-T_{a}^{\infty}(\bar{u})<\varepsilon\right\}\left|T(u) u^{m}-T(\bar{u}) \bar{u}^{m}\right|} \\
& \quad= \begin{cases}\left|u^{m}(u-a)-\bar{u}^{m}(\bar{u}-a)\right| & \text { if } u>a, \bar{u}>a, 0<u-\bar{u}<\varepsilon \\
\left|u^{m}(u-a)\right| & \text { if } u>a, \bar{u}<a, 0<u-a<\varepsilon \\
0 & \text { otherwise. }\end{cases} \\
& \leq C(a) \varepsilon .
\end{aligned}
$$

Therefore, by the coarea formula,

$$
I_{2,2} \leq C(a) \varepsilon \int_{\Omega} \chi_{\left\{0<T_{a}^{\infty}(u)-T_{a}^{\infty}(\bar{u})<\varepsilon\right\}} \mathrm{d}\left|D\left(T_{a}^{\infty}(u)-T_{a}^{\infty}(\bar{u})\right)\right|=\varepsilon o_{\varepsilon}(1)
$$


as $\varepsilon \rightarrow 0$. Combining (5.38), (5.39), (5.40), and (5.41), dividing (5.37) by $\varepsilon$, and passing to the limit as $\varepsilon \rightarrow 0$, we obtain

$$
\begin{aligned}
& \int_{\Omega}((u-f) T(u)-(\bar{u}-\bar{f}) T(\bar{u})) \operatorname{sign}_{0}^{+}\left(T_{a}^{\infty}(u)-T_{a}^{\infty}(\bar{u})\right) \mathrm{d} x \\
& \left.\quad \leq \int_{\Omega}(\overline{\mathbf{z}}, D T(\bar{u}))\right) \\
& \quad+\int_{\partial \Omega} \operatorname{sign}_{0}^{+}\left(T_{a}^{\infty}(u)-T_{a}^{\infty}(\bar{u})\right)(T(u)[\mathbf{z}, \nu]-T(\bar{u})[\overline{\mathbf{z}}, \nu]) d \mathcal{H}^{N-1} .
\end{aligned}
$$

The boundary integral in (5.42) is non-positive: indeed, $T_{a}^{\infty}(u)-T_{a}^{\infty}(\bar{u})>0$ implies $u>a$ and $u>\bar{u}$, and $u>\bar{u}$ implies $u>g$ since $g \leq \bar{g} \leq \bar{u}$. Therefore

$$
\begin{array}{ll}
T(u)[\mathbf{z}, \nu]-T(\bar{u})[\overline{\mathbf{z}}, \nu] & \stackrel{(5.36)}{=} T(u)[\mathbf{z}, \nu] \chi_{\{u>a\}}-T(\bar{u})[\overline{\mathbf{z}}, \nu] \chi_{\{\bar{u}>a\}} \\
\stackrel{(5.4)}{=} & T(u)\left(T_{a}^{\infty}(u)\right)^{m}\left[\mathbf{w} \chi_{\{a<u<b\}}, \nu\right]-T(\bar{u})\left(T_{a}^{\infty}(\bar{u})\right)^{m}\left[\overline{\mathbf{w}} \chi_{\{a<u<b\}}, \nu\right] \\
\stackrel{(5.14 b), g<u}{\leq} & -T(u)\left(T_{a}^{\infty}(u)\right)^{m}+T(\bar{u})\left(T_{a}^{\infty}(\bar{u})\right)^{m} \stackrel{u>\bar{u}}{\leq} 0 .
\end{array}
$$

Hence, dividing (5.42) by $b$ and passing to the limit as $a \rightarrow 0$ and $b \rightarrow 0$ (in this order), we obtain

$$
\int_{\Omega}\left((u-f) \chi_{\{u>0\}}-(\bar{u}-\bar{f}) \chi_{\{\bar{u}>0\}}\right) \operatorname{sign}_{0}^{+}(u-\bar{u}) \mathrm{d} x \leq \lim _{b \rightarrow 0} \frac{1}{b}\left(\lim _{a \rightarrow 0} \int_{\Omega}(\overline{\mathbf{z}}, D T(\bar{u}))\right) .
$$

Let now $\tilde{T}(u)=T_{a}^{b}(u)-b$. We notice that

$$
D \tilde{T}(\bar{u})=D T(\bar{u}), \quad \frac{1}{b} \tilde{T}(s) \stackrel{a \rightarrow 0}{\rightarrow} \frac{1}{b}\left(T_{0}^{b}(s)-b\right) \stackrel{b \rightarrow 0}{\rightarrow}-\chi_{\{s \leq 0\}}
$$

and that

$$
\begin{aligned}
0 \stackrel{(5.15 b)}{\leq} & \int_{\Omega} \mathrm{d}(\overline{\mathbf{z}}, D T(\bar{u})) \stackrel{(5.44)_{1}}{=} \int_{\Omega} \mathrm{d}(\overline{\mathbf{z}}, D \tilde{T}(\bar{u})) \\
\stackrel{(2.4),(5.34)_{2}}{=} & \int_{\partial \Omega} \tilde{T}(\bar{u})[\overline{\mathbf{z}}, \nu] \mathrm{d} \mathcal{H}^{n-1}-\int_{\Omega} \tilde{T}(\bar{u})(\bar{u}-\bar{f}) .
\end{aligned}
$$

Therefore

$$
\begin{aligned}
0 \stackrel{(5.15 b)}{\leq} & \lim _{b \rightarrow 0} \frac{1}{b}\left(\lim _{a \rightarrow 0} \int_{\Omega} \mathrm{d}(\overline{\mathbf{z}}, D T(\bar{u}))\right) \\
\stackrel{(5.44)_{2}}{=} & -\int_{\partial \Omega} \chi_{\{\bar{u}=0\}}[\overline{\mathbf{z}}, \nu] \mathrm{d} \mathcal{H}^{n-1}+\int_{\Omega} \chi_{\{\bar{u}=0\}}(\bar{u}-\bar{f}) \\
\stackrel{(5.6)}{=} & -\int_{\Omega} \chi_{\{\bar{u}=0\}} \bar{f} .
\end{aligned}
$$

Since $\bar{f} \geq 0$, the chain of inequalities in (5.45) implies that

$$
\bar{f}=0 \quad \text { a.e. on }\{\bar{u}=0\} \quad \text { and } \quad 0=\lim _{b \rightarrow 0} \frac{1}{b}\left(\lim _{a \rightarrow 0} \int_{\Omega} \mathrm{d}(\overline{\mathbf{z}}, D T(\bar{u}))\right) .
$$

Analogously, we of course obtain that $f=0$ a.e. on $\{u=0\}$. Therefore (5.43) may be rewritten as

$$
\int_{\Omega}(u-\bar{u}) \operatorname{sign}_{0}^{+}(u-\bar{u}) \mathrm{d} x \leq \int_{\Omega}(f-\bar{f}) \operatorname{sign}_{0}^{+}(u-\bar{u}) \mathrm{d} x \leq \int_{\Omega}(f-\bar{f})^{+} \mathrm{d} x
$$

and the proof is complete. 
Remark 5.12. A supersolution $\bar{u}$ of (1.7) for $m>0$ may be defined as a function which satisfies all properties in Definition 5.4 besides (5.13), which is replaced by

$$
\bar{u}-f \geq \operatorname{div} \overline{\mathbf{z}} \in L^{\infty}(\Omega),
$$

and (5.14b), which is removed. With this definition, the proof of Theorem 5.11 continues to hold and yields $\bar{u} \geq u$. On the other hand, a subsolution $\underline{u}$ of (1.7) may be defined as a function which satisfies all properties in Definition 5.4 besides (5.13), which is replaced by

$$
\underline{u}-f \leq \operatorname{div} \underline{\mathbf{z}} \in L^{\infty}(\Omega),
$$

and $\underline{u} \geq g$, which is removed. With this definition, the proof of Theorem 5.11 (with $u$ replaced by $\underline{u}$ and $\bar{u}$ replaced by $u$ ) continues to hold and yields $\underline{u} \leq u$. Thus, as to the boundary conditions, supersolutions require only that $\bar{u} \geq g$ on $\partial \Omega$, whereas subsolutions require only that (5.14b) holds.

In the singular case $m<0$, analogous considerations lead to suitable definitions of sub and supersolutions for problem (1.7), for which the proof of the comparison principle stated in Theorem 4.8 continues to hold: in this case, supersolutions are only required to satisfy (4.5b), while subsolutions are only required to satisfy $\underline{u} \leq g$ on $\partial \Omega$.

\section{Qualitative properties}

In this section we highlight some qualitative features of solutions to (1.7). Our interest is primarily concerned with their behavior as the boundary value $g$ becomes large. As our analysis is based on comparison, we begin with a few examples of explicit solutions: in particular, constant solutions (which may not attain the boundary values) are given in (i) below; these coincide with solutions with large boundary values for $m<0$, whereas solutions with large boundary values for $m>0$ are given in (ii)-(iv).

Lemma 6.1. Let $\Omega=B_{R}(0)$ for some $R>0$ and let $u$ be the solution to (1.7) with data $f=F \in$ $[0,+\infty)$ and $g=G \in[0,+\infty)$.

(i) If $m<0$, then $u=U$ for all $G \geq U$, where $U \in(0,+\infty)$ is defined by $U-F=U^{m} N / R$. If $m>0$, then $u=U$ for all $G \leq U$, where $U \in[0,+\infty)$ is defined by $U+U^{m} N / R=F$.

(ii) If $0<m<1$ and $G>F$ is sufficiently large, then

$$
u(x)=h(r) \chi_{B_{r}(0)}(\rho)+h(\rho) \chi_{\Omega \backslash B_{r}(0)}(\rho), \quad \rho:=\|x\|,
$$

where $h \in C^{1}([r, R])$, positive and increasing, is the unique solution to

$$
\left\{\begin{array}{l}
m \frac{\mathrm{d} h}{\mathrm{~d} \rho}=h^{1-m}(h-F)-(N-1) \frac{h}{\rho} \\
h(R)=G
\end{array}\right.
$$

and $r \in(0, R)$ is the unique solution to

$$
H_{N}(r):=h(r)-F-\frac{h^{m}(r) N}{r}=0 .
$$


(iii) If $m=1, F=0$, and $R>N$, then

$$
u(x)= \begin{cases}G\left(\frac{R}{N}\right)^{N-1} e^{N-R} & \text { if } \rho<N \\ G\left(\frac{R}{\rho}\right)^{N-1} e^{\rho-R} & \text { if } N<\rho<R .\end{cases}
$$

(iv) If $m>1, F=0$, and $G$ is sufficiently large, then $u=G$.

(v) If $m>1, N=1, F=0$, and $G<\left(\frac{m-1}{m} R\right)^{1 /(m-1)}$ is sufficiently small, then

$$
u(x)=\left(G^{m-1}+\frac{1-m}{m}(R-\rho)\right)_{+}^{\frac{1}{m-1}} .
$$

Proof. Throughout the proof, primes denote differentiation with respect to the radial variable $\rho$. Since all functions $u$ in $(i)-(v)$ are Lipschitz continuous, conditions (4.3) and (5.12) are in fact equivalent to

$$
(\mathbf{w}, D u)=|D u|
$$

(i). If $m<0$, let $u=U$ and $\mathbf{w}(x)=x / R$. Then $\mathbf{z}(x)=U^{m} x / R$ and $\operatorname{div} \mathbf{z}=U^{m} N / R$, so that $u-F=\operatorname{div} \mathbf{z}$ by the choice of $U$. Condition (6.5) is obviously true. Finally, $[\mathbf{z}, \nu]=$ $\frac{U^{m} x}{R} \cdot \frac{x}{R}=U^{m}$, hence the boundary condition holds whenever $G \geq U$. The case $m>0$ is analogous, choosing $\mathbf{w}(x)=-x / R$.

(ii). Recall here $0<m<1$; we look for a solution of the form (6.1) with $0<r<R$ and $h \in C([r, R])$ nonnegative, nondecreasing and such that $G=h(R)$. We define $\mathbf{w}, \mathbf{z} \in X(\Omega)$ by

$$
\mathbf{w}(x):=\left\{\begin{array}{ll}
\frac{x}{r} & \text { if } \rho<r \\
\frac{x}{\rho} & \text { if } \rho>r
\end{array} \quad, \quad \mathbf{z}(x):=u^{m} \mathbf{w}= \begin{cases}\frac{h^{m}(r) x}{r} & \text { if } \rho<r \\
\frac{h^{m}(\rho) x}{\rho} & \text { if } \rho>r .\end{cases}\right.
$$

Then

$$
\operatorname{div} \mathbf{z}= \begin{cases}\frac{h^{m}(r) N}{r} & \text { if } \rho<r \\ m h^{m-1}(\rho) h^{\prime}(\rho)+h^{m}(\rho) \frac{N-1}{\rho} & \text { if } \rho>r\end{cases}
$$

Condition (6.5) holds since

$$
(\mathbf{w}, D u)= \begin{cases}0=|D u| & \text { if } \rho<r \\ \frac{x}{\rho} \cdot h^{\prime}(\rho) \frac{x}{\rho}=h^{\prime}(\rho)=|D u| & \text { if } \rho>r .\end{cases}
$$

The condition $h(R)=G$ in (6.2) implies that $u=g$ on $\partial \Omega$, hence the boundary condition (5.14) holds. The other conditions in (6.2) and (6.3) implies that $\operatorname{div} \mathbf{z}=u-F$. It remains to check that $h$ and $r$ exist and are unique. We discuss the cases $N=1$ and $N>1$ separately.

Case $N=1$. Since $G>F$, (6.2) has a unique solution $h$ in $(-\infty, R]$, with $h$ increasing and $h(\rho) \rightarrow F$ as $\rho \rightarrow-\infty$ (observe that $h$ lies above the stationary solution $F$ ). Since $H_{1}(\rho) \rightarrow-\infty$ 
as $\rho \rightarrow 0^{+}$and $H_{1}(R)>0$ for $G$ sufficiently large (recall that $m<1$ ), (6.3) has a solution. Uniqueness of $r$ will be shown below for any $N \geq 1$.

Case $N>1$. We will argue that there exists a unique solution $h$ to $(6.2)$ in $(0, R]$ with the following properties:

(a) $h^{\prime}(R)>0$

(b) $h>F$ in $(0, R)$;

(c) $h$ has a unique minimum point $\rho_{m} \in(0, R)$.

(a) follows immediately from (6.2) choosing $G$ sufficiently large (in particular, $G>F$ ). (b) follows by contradiction: let $\rho_{0}$ be the closest point to $R$ at which $h(\rho)=F$; if $F>0$, by (6.2) we have $h^{\prime}\left(\rho_{0}\right)<0$ which, together with the fact that $h(R)=G>F$, contradicts the definition of $\rho_{0}$; if $F=0$ then $h$ is identically zero, in contradiction with the condition $h(R)=G$. In order to show $(c)$, assume by contradiction that $h^{\prime}>0$ in $(0, R)$. Then we would have

$$
h^{1-m} \geq h^{-m}(h-F)>\frac{N-1}{\rho} \rightarrow+\infty \quad \text { as } \rho \rightarrow 0^{+},
$$

a contradiction. Therefore at least one point $\rho_{\min } \in(0, R)$ exists with

$$
h^{\prime}\left(\rho_{\text {min }}\right)=0, \text { that is, } h\left(\rho_{\text {min }}\right)-F=\frac{(N-1) h^{m}\left(\rho_{\text {min }}\right)}{\rho_{\min }} .
$$

Differentiating (6.2) and using (6.8), one sees that $h^{\prime \prime}(\rho)>0$ at any point in which $h^{\prime}(\rho)=0$. Therefore $\rho_{\min }$ is unique and $h^{\prime}(\rho)<0$ for $\rho \in\left(0, \rho_{\min }\right)$. Since $H_{N}\left(\rho_{\min }\right)<0$ and $H_{N}(R)>0$ for $G$ sufficiently large (recall that $m<1$ ), there exists $r \in\left(\rho_{\min }, R\right)$ such that $H_{N}(r)=0$.

In order to show now that $r$ (the zero of $H_{N}$ ) is unique, we can reunify the cases $N=1$ and $N \geq 1$. We have that

$$
H_{N}^{\prime}(\rho)=h^{\prime}(\rho)-\frac{N}{\rho} H_{N}(\rho)=H_{N}(\rho)\left(\frac{h^{1-m}}{m}-\frac{N}{\rho}\right)+\frac{h}{m \rho} .
$$

Then, since

$$
H_{N}(\rho) \leq h-\frac{N h^{m}}{\rho}=m h^{m}\left(\frac{h^{1-m}}{m}-\frac{N}{m \rho}\right)<m h^{m}\left(\frac{h^{1-m}}{m}-\frac{N}{\rho}\right),
$$

it holds that

$$
H_{N}^{\prime}(\rho) \geq \frac{h}{m \rho}>0 \quad \text { if } H_{N}(\rho) \geq 0 .
$$

Therefore, there exists a unique $r \in(0, R)$ such that $H_{N}(r)=0$.

(iii). As in (ii), we look for a solution of the form (6.1) with $0<r<R$ and $h \in C([r, R])$ nonnegative, nondecreasing and such that $G=h(R)$. We define w, $\mathbf{z} \in X(\Omega)$ as in (6.6) and, as in (ii), we obtain that $h(r)=\frac{h(r) N}{r}$, i.e. $r=N$, and that $h$ satisfies

$$
h=h^{\prime}+(N-1) \frac{h}{\rho}, \quad N<\rho<R
$$


The solution to (6.9) can be computed explicitly, leading to (6.4). Condition (6.5) holds (cf. (6.7) and note that $h$ is nondecreasing) and $u=G$ on $\partial \Omega$, hence (5.14) holds.

(iv). Let $\mathbf{w}(x)=x \frac{G^{1-m}}{N}$ with $G^{m-1} \geq \frac{R}{N}$, so that $\|\mathbf{w}\|_{\infty} \leq 1$. Then $\mathbf{z}(x)=u^{m}(x) \mathbf{w}(x)=$ $G x$, so that $\operatorname{div} \mathbf{z}=G=u$. Condition (6.5) is obviously true and the boundary datum is attained.

(v). It suffices to define $r=R-\frac{m}{m-1} G^{m-1}$ and $\mathbf{w}, \mathbf{z} \in X(\Omega)$ by

$$
\mathbf{w}(x):=\left\{\begin{array}{ll}
\frac{x}{r} & \text { if } \rho<r \\
\frac{x}{\rho} & \text { if } \rho>r
\end{array} \quad, \quad \mathbf{z}(x):=u^{m} \mathbf{w}= \begin{cases}0 & \text { if } \rho<r \\
h^{m}(\rho) \frac{x}{\rho} & \text { if } \rho>r\end{cases}\right.
$$

and to argue as in item (ii) (with $F=0$ and $N=1$ ).

We now draw a few consequences based on comparison. In the (scaling-wise) linear and superlinear case, $m \geq 1$, solutions blow-up uniformly in the whole domain as the boundary datum becomes large. In particular, no nontrivial large solution can exist.

Proposition 6.2. Let $m \geq 1$ and let $\Omega=B_{R}(0)$ for some $R>0$, with $R>N$ if $m=1$. Let $f \in L^{\infty}(\Omega)$ nonnegative and $g_{G} \in L^{\infty}(\partial \Omega)$ such that $g_{G} \geq G$. Then the solutions $u_{G}$ of (1.7) with data $f$ and $g_{G}$ are such that

$$
u_{G}(x) \rightarrow+\infty \text { for all } x \in B_{R}(0) \text { as } G \rightarrow+\infty .
$$

Proof. In view of the comparison tool given by Theorem 5.11, it suffices to prove the statement for $f=0$ and $g_{G}=G$. In this case solutions are explicitly given by Lemma 6.1(iii)-(iv), whence the result.

On the contrary, in the (scaling-wise) singular case, $m<0$, solutions are bounded independently of their boundary value:

Proposition 6.3. Let $m<0$ and let $\Omega=B_{R}(0)$ for some $R>0$. Let $f \in L^{\infty}(\Omega)$ and let $U$ be defined by $U-\|f\|_{L^{\infty}(\Omega)}=U^{m} N / R$. Then

$$
\|u\|_{L^{\infty}(\Omega)} \leq U
$$

for any nonnegative $g \in L^{\infty}(\partial \Omega)$, where $u$ is the solution $u$ of (1.7) with data $f, g$.

Proof. Let $u_{U}=U$ be the solution to (1.7) with data $f=\|f\|_{L^{\infty}(\Omega)}$ and $g=G$ for all $G \geq U$, as given by Lemma 6.1(i). Then the conclusion follows choosing $G \geq \max \left\{U,\|g\|_{L^{\infty}(\partial \Omega)}\right\}$ and applying the comparison tool given by Theorem 4.8.

The (scaling-wise) sublinear case, $0<m<1$, lies somewhat in between, in the sense that solutions are locally bounded independently of the boundary value $g$.

Proposition 6.4. Let $0<m<1, R>0, \Omega=B_{R}(0), f \in L^{\infty}(\Omega)$. Then

$$
u(x) \leq \bar{u}(\|x\|),
$$

for any nonnegative $g \in L^{\infty}(\partial \Omega)$, where $u$ is the solution of (1.7) with data $f, g$ and

$$
\bar{u}(x):=\bar{h}(r) \chi_{B_{\bar{r}}(0)}(\rho)+\bar{h}(\rho) \chi_{\Omega \backslash B_{\bar{r}}(0)}(\rho), \quad \rho:=\|x\|,
$$


where $\bar{h}=\bar{v}^{\frac{1}{m-1}}, \bar{v}$ is the unique solution to

$$
\frac{\mathrm{d} \bar{v}}{\mathrm{~d} \rho}=\frac{m-1}{m}\left(1-\|f\|_{L^{\infty}(\Omega)} \bar{v}^{1 /(1-m)}-\frac{(N-1) \bar{v}}{\rho}\right), \quad \bar{v}(R)=0
$$

and $\bar{r}$ is the unique solution to $\bar{h}(r)-\|f\|_{L^{\infty}(\Omega)}=\bar{h}^{m}(r) N / r$.

Proof. Let $F=\|f\|_{L^{\infty}(\Omega)}$ and $G \geq\|g\|_{L^{\infty}(\partial \Omega)}$ sufficiently large. We consider the solutions $u$ with data $F, G$ obtained in Lemma 6.1(ii) and index solutions accordingly, i.e. we let $u=u_{G}$, $r=r_{G}$, and $h=h_{G}$. Letting $v_{G}=h_{G}^{m-1}$, we see that $v_{G}$ solves

$$
\frac{\mathrm{d} v_{G}}{\mathrm{~d} \rho}=\frac{m-1}{m}\left(1-F v_{G}^{1 /(1-m)}-\frac{(N-1) v}{\rho}\right), \quad v_{G}(R)=G^{m-1} \stackrel{G \rightarrow+\infty}{\rightarrow} 0 .
$$

Hence, by standard ode theory, $v_{G} \rightarrow \bar{v}$ locally uniformly in $(0, R]$ as $G \rightarrow+\infty$ (in fact, uniformly in $[0, R]$ if $N=1$ ) with $\bar{v}$ as in (6.11), and that $r_{G}$ converges to $\bar{r}$. Finally, it follows from Theorem 5.11 that $u_{G} \geq u$ for all $G$ sufficiently large, hence the result.

Observe that, in Proposition 6.4, one has that $\bar{v} \sim(1-m)(R-\rho) / m$ as $\|x\| \rightarrow R$; therefore,

$$
u \lesssim\left(\frac{1-m}{m}(R-\|x\|)\right)^{\frac{1}{m-1}} \quad \text { as }\|x\| \rightarrow R .
$$

This asymptotic upper bound is optimal, as shown by the following proposition.

Proposition 6.5. Let $0<m<1, R>0, \Omega=B_{R}(0), G>0, f \in L^{\infty}(\Omega)$, and $g=g_{G} \in$ $L^{\infty}(\partial \Omega)$ such that $g_{G} \geq G$. Then the corresponding solutions $u_{G}$ of (1.7) are such that

$$
\liminf _{G \rightarrow \infty} u_{G}(x) \geq \bar{u}_{0}(\|x\|) \quad \text { for all } x \in B_{R}(0),
$$

where $\bar{u}_{0}$ is defined as in (6.10) with $F=0$ in (6.11), and is such that $\bar{u}_{0} \sim\left(\frac{1-m}{m}(R-\|x\|)\right)^{\frac{1}{m-1}}$ as $\|x\| \rightarrow R$.

Proof. In view of the comparison tool given by Theorem (5.11), it suffices to prove the statement for the explicit solutions obtained in Lemma 6.1(ii) with $f=0$ and $g=G$. The proof is identical to the one of the previous Lemma.

Finally, we give two explicit examples of the regularizing effect given by Lemma 4.7: solutions do not jump in the bulk, even if $f$ does.

Example 6.6. Let $R>0, \Omega=B_{R}(0), 0<r<R, f=\alpha \chi_{B_{r}(0)}+\beta \chi_{B_{R}(0) \backslash B_{r}(0)}>0$ $(0<\beta<\alpha)$, and $g=\beta$. Then the solution of (1.7) is $u=\beta$ for all $r$ sufficiently small.

Let again $\rho:=\|x\|$. We choose

$$
\mathbf{w}(x):= \begin{cases}C x & \text { if } \rho \leq r \\ C \frac{r^{N} x}{\rho^{N}} & \text { if } r<\rho<R,\end{cases}
$$

so that $\mathbf{w}$ (hence $\mathbf{z}$ ) is continuous across $\rho=r$ and

$$
\operatorname{div} \mathbf{z}= \begin{cases}\beta^{m} C N & \text { if } \rho \leq r \\ 0 & \text { if } r<\rho<R,\end{cases}
$$


hence $u-f=\operatorname{div} \mathbf{z}$ holds choosing $C=\beta^{-m}(\beta-\alpha) / N$. Finally, imposing $\|\mathbf{w}\|_{\infty} \leq 1$ we obtain

$$
\frac{\alpha-\beta}{N \beta^{m}} r \leq 1 \quad \text { and } \quad \frac{\alpha-\beta}{N \beta^{m}} \frac{r^{N}}{R^{N-1}} \leq 1,
$$

which are satisfied for all $r$ sufficiently small.

Combining this construction with the one in the previous results -through Bernoulli-type equations- one could in fact provide explicit solutions for any $r \in(0, R)$ and any constant boundary value. We give a prototypical example in the special case $m=1, N=1$, where the solution is still explicit.

Example 6.7. Let $N=1, m=1, \Omega=B_{R}(0), 0<r<R, f=\alpha \chi_{B_{r}(0)}+\beta \chi_{\Omega \backslash B_{r}(0)}>0$ $(\alpha>\beta>0)$, and $g=G>0$. Arguing as in Example 6.6, we see that $u=\beta$ is the solution to (1.7) if $G \leq \beta$ and $\frac{\alpha-\beta}{\beta} r \leq 1$. Instead, if $\frac{\alpha-\beta}{\beta} r>1$, we look for solutions of the form

$$
u(x)=A \chi_{B_{r}(0)}+h(\rho) \chi_{\Omega \backslash B_{r}(0)}, \quad \rho:=\|x\|,
$$

for a suitable $A>0$. We choose

$$
\mathbf{w}(x):= \begin{cases}-\frac{x}{r} & \text { if } \rho \leq r \\ -\frac{x}{\rho} & \text { if } r<\rho<R .\end{cases}
$$

By imposing to $u$ to solve problem (1.7) we obtain $A=\alpha\left(\frac{r}{r+1}\right)$ and

$$
-h^{\prime}(\rho)=h(\rho)-\beta \quad \text { for } r<\rho<R .
$$

Integrating and imposing $h(r)=A$, we obtain

$$
h(\rho)=\beta+\left(\frac{\alpha r}{r+1}-\beta\right) e^{r-\rho} .
$$

Observe that the boundary condition is satisfied in the sense of Definition 5.4 as soon as $G \leq \beta$ since $[w, \nu]=-1$ at $\rho=R$.

\section{Homogeneous Neumann boundary conditions and more general nonlinearities}

Existence and uniqueness results analogous to Theorems 4.3 and 5.6 hold for (1.7) with homogenous Neumann boundary conditions. The definition of solution is the following one:

Definition 7.1. Let $f \in L^{\infty}(\Omega)$ be nonnegative with inf $f>0$ if $m<0$. A function $u: \Omega \rightarrow$ $[0,+\infty)$ is a solution of problem (1.11) with datum $f$ if $u \in T B V^{+}(\Omega) \cap L^{\infty}(\Omega)$ and there exists $\mathbf{w} \in L^{\infty}\left(\Omega ; \mathbb{R}^{N}\right)$ such that: $\|\mathbf{w}\|_{\infty} \leq 1, \mathbf{z}:=u^{m} \mathbf{w} \in X(\Omega)$ satisfies

$$
\begin{gathered}
\left|D \phi\left(T_{a}^{b}(u)\right)\right| \leq\left(\mathbf{z}, D T_{a}^{b}(u)\right) \quad \text { as measures for a.e. } 0<a<b \leq+\infty, \\
u-f=\operatorname{div} \mathbf{z} \quad \text { in } \mathcal{D}^{\prime}(\Omega),
\end{gathered}
$$

and

$$
[\mathbf{z}, \nu]=0 \quad \mathcal{H}^{N-1} \text {-a.e. on } \partial \Omega .
$$


Theorem 7.2. Let $f \in L^{\infty}(\Omega)$ be nonnegative with inf $f>0$ if $m<0$. Then there exists a unique solution $u$ of (1.11) with datum $f$ in the sense of Definition 7.1. In addition, $u \in D T B V^{+}(\Omega)$,

$$
\left(\mathbf{w}, D T_{a}^{b}(u)\right)=\left|D T_{a}^{b}(u)\right| \text { for a.e. } 0<a<b \leq+\infty,
$$

and

$$
\left(\mathbf{z}, D T_{a}^{b}(u)\right)=\left|D \phi\left(T_{a}^{b}(u)\right)\right| \quad \text { for a.e. } 0<a<b \leq+\infty .
$$

Sketch of the proof. The proof of Theorem 7.2 closely follows the lines of that of Theorems 4.3, $4.8,5.6$, and 5.11, with many simplifications due to the homogeneous Neumann boundary conditions. We only mention that one has to use the following approximating problems:

$$
\begin{cases}u_{\varepsilon}-f=\operatorname{div}\left(\left(\varepsilon+\left|u_{\varepsilon}\right|\right)^{m} \frac{\nabla u_{\varepsilon}}{\left|\nabla u_{\varepsilon}\right|_{\varepsilon}}+\varepsilon \nabla u_{\varepsilon}\right) & \text { in } \Omega \\ \left(\left(\varepsilon+\left|u_{\varepsilon}\right|\right)^{m} \frac{\nabla u_{\varepsilon}}{\left|\nabla u_{\varepsilon}\right|_{\varepsilon}}+\varepsilon \nabla u_{\varepsilon}\right) \cdot \nu=0 & \text { on } \partial \Omega,\end{cases}
$$

whose solutions $u_{\varepsilon}$ satisfy

$$
\inf f \leq u_{\varepsilon} \leq\|f\|_{L^{\infty}(\Omega)} .
$$

The estimates and the passage to the limit in $\Omega$ are completely analogous, in fact simpler, due to the absence of boundary terms: for instance, in the proof of Lemma 4.5 one has to use lower semi-continuity of the functional

$$
u \in L^{1}(\Omega) \mapsto\left\{\begin{array}{cc}
\int_{\Omega} \varphi d\left|D \phi_{F}(u)\right| & \text { if } u \in B V(\Omega) \\
+\infty & \text { otherwise }
\end{array}, \text { with } 0 \leq \varphi \in \mathcal{D}(\Omega)\right.
$$

(see [1, Theorem 3.1]) which does not contain any boundary contribution. The boundary condition (7.3) can be shown to hold as follows. The fluxes

$$
\mathbf{z}_{\varepsilon}:=\left(\varepsilon+\left|u_{\varepsilon}\right|\right)^{m} \frac{\nabla u_{\varepsilon}}{\left|\nabla u_{\varepsilon}\right|_{\varepsilon}}+\varepsilon \nabla u_{\varepsilon}
$$

satisfy (in view of (2.5) and since $\left[\mathbf{z}_{\varepsilon}, \nu\right]=0$ on $\partial \Omega$ )

$$
0=\int_{\Omega} \varphi \operatorname{div} \mathbf{z}_{\varepsilon}-\int_{\Omega} \mathbf{z}_{\varepsilon} \cdot \nabla \varphi \quad \text { for all } \varphi \in C^{\infty}(\bar{\Omega})
$$

and are such that $\mathbf{z}_{\varepsilon} \rightarrow \mathbf{z}$ in $L^{2}\left(\Omega ; \mathbb{R}^{N}\right)$ and $\operatorname{div} \mathbf{z}_{\varepsilon} \stackrel{*}{\rightarrow} \operatorname{div} \mathbf{z}$ in $\mathcal{M}(\Omega)$. Hence, passing to the limit as $\varepsilon \rightarrow 0$ in (7.5) we obtain

$$
0=\int_{\Omega} \varphi \operatorname{div} \mathbf{z}-\int_{\Omega} \mathbf{z} \cdot \nabla \varphi \stackrel{(2.5)}{=} \int_{\partial \Omega} \varphi[\mathbf{z}, \nu] \mathrm{d} \mathcal{H}^{N-1},
$$

for all $\varphi \in C^{\infty}(\bar{\Omega})$, implying that $[\mathbf{z}, \nu]=0$ on $\partial \Omega$.

Remark 7.3. The arguments in Lemmas 4.7 and 5.9, leading to a null singular set, apply also to the resolvent equation of other parabolic equations with linear growth lagrangian, equations, such that of the relativistic heat equation $(m=1)$ and the relativistic porous medium equation $(m>1$, cf. (1.4)),

$$
u-f=\operatorname{div}\left(\frac{|u|^{m} \nabla u}{\sqrt{u^{2}+|\nabla u|^{2}}}\right) \quad \text { for } m \geq 1,
$$


or that of the speed-limited porous medium equation (cf. (1.6)),

$$
u-f=\operatorname{div}\left(\frac{|u| \nabla u^{M-1}}{\sqrt{1+\left|\nabla u^{M-1}\right|^{2}}}\right) \quad \text { for } M>1,
$$

studied in $[4,8,20,18]$ under different types of boundary conditions (compare condition (5.28) with (3.26) in [4], (34) in [8], (50) in [20], and condition 3 of Definition 8.3 in [18]). Therefore, the unique solutions of those problems belong as well to $D T B V^{+}(\Omega)$. Note, however, that the proof of Lemmas 4.7 and 5.9 does not carry over to $m=0$, where indeed solutions may have jumps.

Remark 7.4. Throughout the paper, we have focused on the case of a mobility given by the nonlinear term $u^{m}$. However, the proofs of both existence and uniqueness of solutions for both problem (1.7) and problem (1.11) still hold in the case of a more general nonlinearity:

$$
\begin{cases}u-f=\operatorname{div}\left(\phi^{\prime}(u) \frac{\nabla u}{|\nabla u|}\right) & \text { in } \Omega \\ u=g & \text { on } \partial \Omega\end{cases}
$$

(we use $\phi^{\prime}$ for consistency with (2.2)), where either

(S) $\phi^{\prime}(s)$ is a locally continuous strictly decreasing function on $(0,+\infty)$

or

(D) $\phi^{\prime}(s) \in C([0,+\infty))$ is a strictly increasing function.

Of course, (S) and (D) represent the singular case $(m<0)$ and the degenerate case $(m>0)$ of the previous sections, respectively. The respective assumptions on $f$ and $g$ are identical (for instance, in case $(\mathrm{S})$ one asks that $g$ be strictly positive on $\partial \Omega$ ). Definitions 4.1, respectively 5.4 , can be modified accordingly, by formally substituting $u^{m}$ with $\phi^{\prime}(u)$.

Acknowledgments. The second and third author acknowledge partial support by the Spanish MEC and FEDER project MTM2015-70227-P. The third author has been partially supported by the Gruppo Nazionale per l'Analisi Matematica, la Probabilità e le loro Applicazioni (GNAMPA) of the Istituto Nazionale di Alta Matematica (INdAM).

\section{References}

[1] M. Amar, V. De Cicco, and N. Fusco. A relaxation result in BV for integral functionals with discontinuous integrands. ESAIM Control Optim. Calc. Var., 13(2):396-412, 2007.

[2] L. Ambrosio, G. Crippa, and S. Maniglia. Traces and fine properties of a $B D$ class of vector fields and applications. Ann. Fac. Sci. Toulouse Math. (6), 14(4):527-561, 2005.

[3] L. Ambrosio, N. Fusco, and D. Pallara. Functions of bounded variation and free discontinuity problems. Oxford Mathematical Monographs. The Clarendon Press, Oxford University Press, New York, 2000.

[4] F. Andreu, V. Caselles, and J. M. Mazón. A strongly degenerate quasilinear elliptic equation. Nonlinear Anal., 61(4):637-669, 2005.

[5] F. Andreu, V. Caselles, and J. M. Mazón. A strongly degenerate quasilinear equation: the parabolic case. Arch. Ration. Mech. Anal., 176(3):415-453, 2005.

[6] F. Andreu, V. Caselles, and J. M. Mazón. Some regularity results on the 'relativistic' heat equation. J. Differential Equations, 245(12):3639-3663, 2008.

[7] F. Andreu, V. Caselles, J. M. Mazón, and J. S. Moll. A diffusion equation in transparent media. J. Evol. Equ., 7(1):113-143, 2007. 
[8] F. Andreu, V. Caselles, J. M. Mazón, and Salvador Moll. The Dirichlet problem associated to the relativistic heat equation. Math. Ann., 347(1):135-199, 2010.

[9] F. Andreu, V. Caselles, J. M. Mazón, J. Soler, and M. Verbeni. Radially symmetric solutions of a tempered diffusion equation. A porous media, flux-limited case. SIAM J. Math. Anal., 44(2):1019-1049, 2012.

[10] F. Andreu-Vaillo, V. Caselles, and J. M. Mazón. Parabolic quasilinear equations minimizing linear growth functionals, volume 223 of Progress in Mathematics. Birkhäuser Verlag, Basel, 2004.

[11] G. Anzellotti. Pairings between measures and bounded functions and compensated compactness. Ann. Mat. Pura Appl. (4), 135:293-318 (1984), 1983.

[12] M. Bertsch and R. Dal Passo. Hyperbolic phenomena in a strongly degenerate parabolic equation. Arch. Rational Mech. Anal., 117(4):349-387, 1992.

[13] P. Blanc. On the regularity of the solutions of some degenerate parabolic equations. Comm. Partial Differential Equations, 18(5-6):821-846, 1993.

[14] F. E. Browder. Pseudo-monotone operators and nonlinear elliptic boundary value problems on unbounded domains. Proc. Nat. Acad. Sci. U.S.A., 74(7):2659-2661, 1977.

[15] J. Calvo. Analysis of a class of degenerate parabolic equations with saturation mechanisms. SIAM J. Math. Anal., 47(4):2917-2951, 2015.

[16] J. Calvo, J. Campos, V. Caselles, O. Sánchez, and J. Soler. Pattern formation in a flux limited reaction-diffusion equation of porous media type. Invent. Math., 206(1):57-108, 2016.

[17] J. Calvo, J. Campos, V Caselles, O. Snchez, and J Soler. Qualitative behavior for flux-saturated mechanisms: Travelling waves and smoothing effects. Journal of the European Mathematical Society, to appear.

[18] J. Calvo and V. Caselles. Local-in-time regularity results for some flux-limited diffusion equations of porous media type. Nonlinear Anal., 93:236-272, 2013.

[19] J. A. Carrillo, V. Caselles, and S. Moll. On the relativistic heat equation in one space dimension. Proc. Lond. Math. Soc. (3), 107(6):1395-1423, 2013.

[20] V. Caselles. On the entropy conditions for some flux limited diffusion equations. J. Differential Equations, 250(8):3311-3348, 2011.

[21] G-Q Chen and H. Frid. Divergence-measure fields and hyperbolic conservation laws. Arch. Ration. Mech. Anal., 147(2):89-118, 1999.

[22] L. Giacomelli. Finite speed of propagation and waiting time phenomena for degenerate parabolic equations with linear growth Lagrangian. SIAM J. Math. Anal., 47(3):2426-2441, 2015.

[23] L. Giacomelli, S. Moll, and F. Petitta. Optimal waiting time bounds for flux-saturated diffusion equations. Comm. Partial Differential Equations, accepted.

[24] M. Latorre and S. Segura de León. Existene and comparison results for an elliptic equations involving the 1laplacian and 11-data. preprint.

[25] J. M. Mazón and S. Segura de León. The Dirichlet problem for a singular elliptic equation arising in the level set formulation of the inverse mean curvature flow. Adv. Calc. Var., 6(2):123-164, 2013.

[26] S. Moll and F. Petitta. Large solutions for nonlinear parabolic equations without absorption terms. J. Funct. Anal., 262(4):1566-1602, 2012.

[27] S. Moll and F. Petitta. Large solutions for the elliptic 1-Laplacian with absorption. J. Anal. Math., 125:113-138, 2015.

[28] P Rosenau. Tempered diffusion: A transport process with propagating front and inertial delay. Phys. Rev A, 146:7371-7374, 1992.

[29] G. Sapiro. Geometric partial differential equations and image analysis. Cambridge University Press, Cambridge, 2006.

[30] R. E. Showalter. Monotone operators in Banach space and nonlinear partial differential equations, volume 49 of Mathematical Surveys and Monographs. American Mathematical Society, Providence, RI, 1997. 